\title{
Protocol-Based Tobit Kalman Filter under Integral Measurements and Probabilistic Sensor Failures
}

\author{
Hang Geng, Zidong Wang, Lei Zou, Alireza Mousavi and Yuhua Cheng
}

\begin{abstract}
This paper is concerned with the Tobit Kalman filtering problem for a class of discrete time-varying systems subject to censored observations, integral measurements and probabilistic sensor failures under the Round-Robin protocol (RRP). The censored observations are characterized by the Tobit observation model, the integral measurements are described as functions of system states over a certain time interval required for data acquisition, and the sensor failures are governed by a set of uncorrelated random variables. The RRP is employed to decide the transmission sequence of sensors in order to alleviate undesirable data collisions. By resorting to the augmentation technique and the orthogonality projection principle, a protocolbased Tobit Kalman filter (TKF) is developed with the coexistence of integral measurements and sensor failures that lead to a couple of augmentation-induced terms. Moreover, the performance of the proposed filter is analyzed through examining the statistical property of the error covariance of the state estimation. Further analysis shows the existence of self-propagating upper and lower bounds on the estimation error covariance. A case study on ballistic roll rate estimation is presented to illustrate the efficacy of the developed filter.
\end{abstract}

Index Terms-Censored observations, integral measurements, Round-Robin protocol, sensor failures, Tobit Kalman filtering.

\section{INTRODUCTION}

Measurement censoring is a particular form of measurement nonlinearity, in which the sensor output is a continuous function of the system state within a certain dynamic range and is constant outside this range [2], [25]. The phenomenon of measurement censoring is often caused by the saturation of sensor outputs as a result of dynamic changes or interferences [4], [42]. Many estimation applications, especially those massively using low-cost commercial off-the-shelf sensors (e.g. pose estimation [1], decentralized detection [4] and optical transmission [18]), are ubiquitously confronted by censored observations, and this has triggered persistent research interest on state estimation problems with measurement censoring in the last few years.

In case of censored observations, the conventional Kalman filter $(\mathrm{KF})$ becomes futile since the measurement noises $\mathrm{n}$ earing the censoring region turn out to be non-Gaussian with

This work is supported in part by the National Natural Science Foundation of China under Grants 61803074, 61703245, U2030205, 61903065, 61671109, U1830207 and U1830133, the China Postdoctoral Science Foundation under Grants 2018T110702, 2018M643441, 2017M623005 and 2015M5825, the Royal Society of the UK, and the Alexander von Humboldt Foundation of Germany. (Corresponding author: Yuhua Cheng.)

H. Geng and Y. Cheng are with the School of Automation Engineering, University of Electronic Science and Technology of China, Chengdu 611731, P. R. China. (Email: yhcheng@uestc.edu.cn)

Z. Wang, L. Zou and A. Mousavi are with the Department of Computer Science, Brunel University London, Uxbridge, Middlesex, UB8 3PH, United Kingdom. (Email: Zidong.Wang@brunel.ac.uk) unknown statistics [2], [7]. As such, direct employment of the standard KF would lead to biased estimates or even degraded performances because of the violation of the Gaussian noise assumption [5], [11], [26], [28]. To handle this issue, enormous endeavors have been made in the literature, where the overwhelming majority build themselves on the existing estimation techniques. Among various filters proposed so far, we highlight the iterative KF [39], particle filter [24] and TKF [2] that have proven to be rather popular.

Since the pioneering work in [2], the so-called TKF has proven to be a powerful approach of practical significance in dealing with censored observations. By introducing new definitions (of the measurement expectation, residual as well as variance) and exploiting a local approximation in calculating censoring probabilities, the TKF is capable of formalizing a fully recursive state estimation paradigm to handle the measurement nonlinearity caused by censored observations. Due to its succinct structure and recursive form, much research enthusiasm has recently been attracted towards the TKF and a number of excellent results have been acquired with successful applications in cooperative localization, fault detection, target tracking, and so forth [12], [21], [23]. Within the TKF framework, the state estimation problem has been tackled in [23] with both censored observations and time-correlated multiplicative noises. Later, TKFs under modelling uncertainties, non-Gaussian noises, redundant channel transmission and packet delays have been developed, respectively, in [12], [14]-[16] with applications to ballistic roll rate tracking and maneuvering target tracking problems. In addition, the fault detection problem has been solved in [21] via a variant TKF approach for discrete-time linear systems with dead-zone-like censoring.

In the context of state estimation problems, the overwhelming majority of the existing work has implicitly assumed that the sensor observation relies only on the current system state [19], [31]-[35]. This assumption is, unfortunately, sometimes unreasonable in certain applications such as the chemical reaction, nuclear fusion and synchrotron radiation [6], [36], [43]. In these applications, the sensor observation is actually proportional to so-called integral measurements [13], that is, the integral of system states within a prescribed time interval due presumably to delayed data acquisition and analysis. In [17], a novel characterization in relation to the integral measurements has been proposed where the measurement output has been described as a function of the system states over a given period in response to the time slot required for the sample collection. Then, a modified unscented KF has been developed to accomplish the task of state estimation for the 
concerned nonlinear systems. Taking use of such a novel characterization, the simultaneous state and fault reconstruction problem with integral measurements and ambient disturbances has been addressed in [30] by referencing to the augmentation technique and the unknown input observer approach.

Another underlying assumption behind the conventional estimation schemes is that the sensors are equipped with the capability to provide accurate observations to the designed estimator. This assumption, however, does not always hold in reality. More often than not, sensors working in real-world circumstances are ineluctably confronted with all sorts of failures due mainly to abrupt environment changes, unexpected exogenous disturbances, internal component ageing and so forth (see [22], [29], [37], [45] and the references therein). Basically speaking, sensor failures usually happen in a probabilistic way which result in the phenomenon of measurement missing/degradation. The seminal work on filtering problems with measurement missing/degradation can be dated back to 1969 in reference [37], where an optimal recursive filter has been put forward for systems suffering from missing measurements [37]. In [45], the variance-constrained state estimation problem has been coped with for multi-rate systems susceptible to quantized and degraded observations in terms of stochastic analysis techniques. Recently, regarding the constrained network resource, an event-triggered resilient filter has been designed in [27] for systems subject to the simultaneous presence of measurement quantization and sensor failures, where the variation of the filter gain has also been taken into account.

In the past few decades, the networked system has gained a surge of research attention due largely to its broad applications in industrial fabrication, environmental monitoring and target localization, see e.g. [27], [40], [41], [47], [48]. In an ideal situation, all system components (e.g. actuators, controllers, filters and sensors) are supposed to have privileges for information propagation via shared communication networks. This supposition, however, is often impractical as limitedbandwidth-induced data collisions are likely to happen when the information exchanges take place simultaneously by more than one component [9], [46]. In this respect, communication protocols have been leveraged to orchestrate the transmission sequence of system components by giving the transmission permission to a single component at each time instant so as to avoid possible data collisions (see [41], [48], [49] and the references therein). Among the various communication protocols, the Round-Robin protocol (RRP) has drawn particular research attention because of its succinct execution manner, where the information propagation among system components is conducted in a fixed circular order [44]. Recently, the distributed set-membership filtering problem has been solved in [29] for a class of multi-rate systems in sensor networks under the RRP scheduling, where the desired filter gains have been obtained by minimizing a certain ellipsoid in the sense of the minimum traces of weighted matrices.

To conclude the above discussions, we make the following observations: 1) although much work has been done hitherto on the design of TKFs under different circumstances, there has been a lack of analysis results on the corresponding filtering performance within a holistic Tobit Kalman filtering framework; 2) due to unreliable working conditions and delayed data acquisition and analysis, sensors are inclined to experience probabilistic failures and integral measurements, and the negligence of such phenomena would result in deteriorated filtering performances; 3 ) communication protocols have proven to be beneficial in boosting transmission scheduling and circumventing data collisions, but the investigation into TKFs under communication protocols has been still in its infancy due mainly to the difficulty of appropriately describing TKF-embedded protocol characteristics.

Following the observations made previously, a seemingly natural research topic is to devise a protocol-based TKF, in the presence of sensor outputs undergoing integral measurements and sensor failures, to achieve the optimal state estimation and also evaluate the associated filtering performance. This topic, though theoretically important and practically significant, is quite challenging for three reasons: 1) it is unclear as how to derive a protocol-based Tobit regression model in conjunction with integral measurements and sensor failures; 2 ) it is fairly difficult to conduct the performance analysis on the developed filter due to its time-varying and stochastic nature; 3) it is mathematically hard to examine the joint impacts from the communication protocol, integral measurements and sensor failures on the design and performance analysis of the filter. Therefore, the main purpose of this paper is to overcome the identified challenges.

In this paper, we endeavor to deal with the protocolbased Tobit Kalman filter under integral measurements and probabilistic sensor failures. To be more specific, a protocolbased Tobit regression model is first built that accommodates the integral measurements and randomly occurring sensor failures. By resorting to the orthogonality projection principle, an optimal protocol-based TKF is designed in the sense of linear minimum mean-squared error (LMMSE), where most of its computation can be carried out recursively or off-line. In addition, the performance of the desired filter is statistically assessed, and sufficient conditions are established for the existence of self-propagating upper and lower bounds on the estimation error covariance.

The main contributions are highlighted as follows. i) To our knowledge, this paper presents one of the first few attempts to look into the Tobit Kalman filtering problem with integral measurements and sensor failures under the communication protocol, where the system model is both holistic and comprehensive in catering for engineering practice. ii) Compared with the TKF in [2], a couple of new terms emerge in the newly designed filter, which is seen as an envisioned reflection of the addressed communication protocol, integral measurements and sensor failures. iii) The performance of the developed filter is evaluated via analyzing the estimation error covariance with its upper and lower bounds that are found to be selfpropagating.

The remainder of the paper is organized as follows. In Section II, the problem under consideration is formulated. In Section III, an optimal protocol-based TKF is designed. In Section IV, the performance of the developed filter is evaluated. In Section V, a numerical example is provided to 


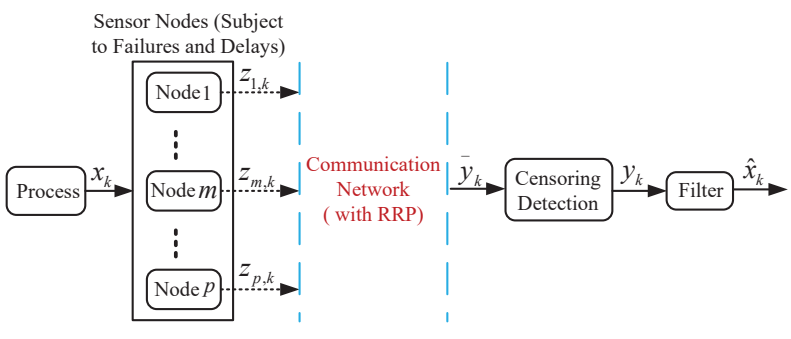

Fig. 1: Schematic diagram for the concerned Tobit Kalman filtering problem.

show the usefulness of the filter, and some conclusions are drawn in Section VI.

Notation The notation used here is fairly standard except where otherwise stated. $\mathbb{R}^{n}$ denotes the $n$-dimensional Euclidean space. "I" and "0" represent identity and zero matrices with proper dimensions, respectively. Superscripts " -1 " and " $T$ " represent inverse and transpose operations, respectively. $\mathbb{E}\{x\}$ and $\mathbb{E}\{x \mid y\}$ denote the expectation of $x$ and the expectation of $x$ conditional on $y$, respectively. $\operatorname{var}\{X\}$ stands for the variance of $X \cdot \operatorname{tr}\{X\}$ represents the trace of matrix $X . \delta(\cdot) \in\{0,1\}$ is the Dirac delta function.

\section{PRoblem Formulation}

Consider the Tobit Kalman filtering problem for a networked system as shown in Fig. 1. In this framework, the sensor is susceptible to probabilistic failures, the sampling is subject to delays, the signal transmission between the filter and the sensor is implemented through a communication network under the RRP, and the measurement arriving at the filter is inclined to censoring. In what follows, let us introduce the plant, the communication network, and the degraded, integral and censored measurement in a mathematical way.

Consider the following linear discrete time-varying system subject to integral measurements [17], [30] and probabilistic sensor failures [27], [45]:

$$
\begin{aligned}
x_{k+1} & =A_{k} x_{k}+\omega_{k}, \\
z_{m, k} & =\Lambda_{m, k} C_{m, k} \sum_{s=0}^{\ell} x_{k-s}+v_{m, k}, m=1,2, \ldots, p,
\end{aligned}
$$

where $x_{k} \in \mathbb{R}^{n_{x}}$ is the state vector and $z_{m, k} \in \mathbb{R}$ is the uncensored observation of the $m$ th sensor. $A_{k}$ and $C_{m, k}$ are known time-varying matrices with compatible dimensions. $\Lambda_{m, k} \in \mathbb{R}$ is the sensor failure coefficient, $\ell$ is the time length required for the data collection, and $p$ is the number of sensors. $\omega_{k} \in \mathbb{R}^{n_{x}}$ and $v_{m, k} \in \mathbb{R}$ are zero-mean white Gaussian noises with covariances $Q_{k}$ and $R_{m, k}$, respectively.

In the current investigation, the sensor measurements $z_{m, k}$ $(m=1,2, \ldots, p)$ are transmitted to the remote estimator via a shared communication network. Due to limited communication bandwidth, it is assumed that at each communication time instant, only one single sensor is granted the access to the shared channel to transmit its output through the network. Accordingly, the RRP is leveraged to orchestrate the transmission order of the sensors with a view to avoiding data collisions.
Define $\hbar_{k} \triangleq \bmod (k-1, p)+1 \in\{1,2, \ldots, p\}$ as the selected sensor that has access to the network at time $k$ where $\bmod (k-1, p)$ is the unique non-negative remainder on division of $k-1$ by $p$, and $\Gamma_{m, \hbar_{k}} \triangleq \delta\left(\hbar_{k}-m\right)$ as the measurement update matrix that regulates the token-dependent scheduling of the $m$ th sensor. Under the RRP and the zero-input strategy, the actual measurement that is sent to the estimator at time $k$ is [29], [48]:

$$
\bar{y}_{k}=\sum_{m=1}^{p} \Gamma_{m, \hbar_{k}} z_{m, k} .
$$

At the input terminal of the estimator, let an additional detection device be equipped to check whether the received $\bar{y}_{k}$ is censored or not, and this gives rise to the following Tobit observation model [2]:

$$
y_{k}=\left\{\begin{array}{cc}
\bar{y}_{k}, & \bar{y}_{k}>\tau \\
\tau, & \bar{y}_{k} \leq \tau
\end{array}\right.
$$

where $y_{k} \in \mathbb{R}$ is the censored observation with a constant threshold $\tau$.

Based on the above Tobit observation model (4), let us define a Bernoulli random variable $\gamma_{k}$ to regulate the censoring phenomenon of $y_{k}$ as follows:

$$
\gamma_{k}= \begin{cases}1, & \bar{y}_{k}>\tau, \\ 0, & \bar{y}_{k} \leq \tau,\end{cases}
$$

with the following probability distribution:

$$
\operatorname{Prob}\left\{\gamma_{k}=1\right\}=\bar{\gamma}_{k}, \operatorname{Prob}\left\{\gamma_{k}=0\right\}=1-\bar{\gamma}_{k} .
$$

Here, $\bar{\gamma}_{k}$ is a known non-negative constant. It is supposed that $\gamma_{k}$ is uncorrelated with other noise signals. Taking advantage of $\gamma_{k}, y_{k}$ in (4) can be rewritten as follows:

$$
y_{k}=\gamma_{k} \bar{y}_{k}+\left(1-\gamma_{k}\right) \tau \text {. }
$$

Let

$$
y_{1: k} \triangleq\left\{y_{1}, y_{2}, \ldots, y_{k}\right\}, \quad \gamma_{1: k} \triangleq\left\{\gamma_{1}, \gamma_{2}, \ldots, \gamma_{k}\right\}
$$

be the measurement and censoring sequences up till time $k$, respectively. Furthermore, we denote

$$
\begin{aligned}
& \hat{x}_{k}^{-} \triangleq \mathbb{E}\left\{x_{k} \mid y_{1: k-1}, \gamma_{1: k-1}\right\}, \quad \tilde{x}_{k}^{-} \triangleq x_{k}-\hat{x}_{k}^{-}, \\
& \hat{x}_{k} \triangleq \mathbb{E}\left\{x_{k} \mid y_{1: k}, \gamma_{1: k}\right\}, \quad \tilde{x}_{k} \triangleq x_{k}-\hat{x}_{k}, \\
& \hat{y}_{k}^{-} \triangleq \mathbb{E}\left\{y_{k} \mid y_{1: k-1}, \gamma_{1: k-1}\right\}, \quad \tilde{y}_{k}^{-} \triangleq y_{k}-\hat{y}_{k}^{-}, \\
& P_{\tilde{x}_{k}} \triangleq \mathbb{E}\left\{\tilde{x}_{k} \tilde{x}_{k}^{T} \mid y_{1: k}, \gamma_{1: k}\right\}, \\
& P_{\tilde{y}_{k}^{-}} \triangleq \mathbb{E}\left\{\tilde{y}_{k}^{-}\left(\tilde{y}_{k}^{-}\right)^{T} \mid y_{1: k-1}, \gamma_{1: k-1}\right\}, \\
& P_{\tilde{x}_{k}^{-}} \triangleq \mathbb{E}\left\{\tilde{x}_{k}^{-}\left(\tilde{x}_{k}^{-}\right)^{T} \mid y_{1: k-1}, \gamma_{1: k-1}\right\}, \\
& P_{\tilde{x}_{k}^{-} \tilde{y}_{k}^{-}} \triangleq \mathbb{E}\left\{\tilde{x}_{k}^{-}\left(\tilde{y}_{k}^{-}\right)^{T} \mid y_{1: k-1}, \gamma_{1: k-1}\right\} .
\end{aligned}
$$

Assumption 1: The initial state $x_{0}$ has the mean $\bar{x}_{0}$ and covariance $P_{0}$. The random variables $x_{0}, \Lambda_{m, k}, \omega_{k}$ and $v_{m, k}$ are mutually independent.

Assumption 2: The sensor failure coefficients $\Lambda_{m, k}(m=$ $1,2 \ldots, p)$ are mutually independent random variables in $m$ and $k$, and are also uncorrelated with $\gamma_{k}$ and other noise signals. $\Lambda_{m, k}$ regulate the probabilistic failure phenomena of 
the $m$ th sensor at time $k$ and take values on the interval $[0,1]$ with certain probability density functions (PDFs) of means $\bar{\Lambda}_{m, k}$ and variances $\breve{\Lambda}_{m, k}$.

Remark 1: It is noteworthy that the measurement sequence $y_{1: k}$ relies on the random censoring sequence $\gamma_{1: k}$, implying that $y_{1: k}$ contains information of $\gamma_{1: k}$, and $y_{1: k}$ will be different for different realizations of $\gamma_{1: k}$. As a result, all expectations defined above are conditional expectations in regard to $\gamma_{1: k}$. Thus, in this paper, we are interested in the statistical property of the error covariance $P_{\tilde{x}_{k}^{-}}$.

When designing filtering algorithms, a widely accepted assumption is that the current sensor observation depends merely on the current state, whereas past states do exert influence on the current observation in the event that a time interval is required for data acquisition and analysis. Hence, the observation in (2) is modeled as the integral of states over a prescribed time slot to characterize such influence. A typical example of the integral measurement can be found in distillation columns [17], where lab analysis is often required for measurements of the distillate and bottom compositions as the use of online analyzers is often infeasible due to economic considerations or technological difficulties. In order to analyze the composition, a sufficient amount of samples must be collected and the sample collection process cannot be completed instantaneously but in a time interval. Accordingly, the measurement model for the sampled distillate and bottom compositions can be written as (2), where $x_{k}$ is the state of distillate and bottom compositions, $\ell$ is the time interval required to complete the sample collection, $C_{m, k}$ are the known measurement matrices of the lab analysis, and $v_{m, k}$ are the lab analysis errors which are assumed to be zero-mean white Gaussian noises with covariances $R_{m, k}$.

Remark 2: It is worth noting that, model (2) is comprehensive as it accounts for several frequently encountered measurement uncertainties (e.g. the packet dropout, time delay and measurement degradation). Specifically, at time $k$, if $\Lambda_{m, k}=0$, it is implied that the $m$ th sensor suffers from the entire failure and its output signa is completely missing; if $\Lambda_{m, k}=1$ and $\ell=0$, it is implied that the $m$ th sensor works in a good condition and no delayed sample collections exist; if $\Lambda_{m, k}=1$ and $\ell>0$, it is implied that though the $m$ th sensor functions well, its sample collection is delayed by a time interval $\ell$; if $0<\Lambda_{m, k}<1$ and $\ell=0$, it is implied that the $m$ th sensor undergoes partial failures and its output signal is measured with reduced gains that lead to degraded measurements; if $0<\Lambda_{m, k}<1$ and $\ell>0$, it is implied that the $m$ th sensor is susceptible to both failures and delayed sample collections, yielding the integral and degraded observations.

As an efficient tool for handling censored observations, the TKF has stirred much research interest during the last few years. By bringing in new definitions (of the measurement expectation, residual as well as variance), the TKF is capable of formalizing a fully recursive state estimation paradigm to process the uncertainty caused by censored observations. Apart from the measurement censoring, sensor outputs are easily prone to uncertainties ranging from integral measurements to probabilistic sensor failures as shown in (2), and the corre- sponding Tobit Kalman filtering problem has not yet been fully investigated, let alone the case where the RRP is employed to reinforce the reliability of network communication. As such, there is a practical need to establish a holistic protocol-based Tobit Kalman filtering framework to fill in such a gap.

It is observed from (4) that, the random variable $\gamma_{k}$ is employed to describe the censoring phenomenon of $y_{k}$. In accordance with (4), if no censoring occurs for $y_{k}$, i.e. $\gamma_{k}=1$, the measurement becomes $y_{k}=\bar{y}_{k}$, which means that the output observation is equivalent to the latent one. If the censoring occurs for $y_{k}$, i.e. $\gamma_{k}=0$, the measurement becomes $y_{k}=\tau$, which means that the censoring threshold is allocated to the output observation. Here, we suppose that the censoring probability $\bar{\gamma}_{k}$ is known a priori via some statistical experiments. Alternatively, inspired by [2], $\bar{\gamma}_{k}$ can also be approximated by

$$
\bar{\gamma}_{k} \approx \Phi\left(\frac{\sum_{m=1}^{p} \sum_{s=0}^{\ell} \Gamma_{m, \hbar_{k}} \bar{\Lambda}_{m, k} C_{m, k} \varsigma_{k-s}-\tau}{\sqrt{\sum_{m=1}^{p} \Gamma_{m, \hbar_{k}}^{2} R_{m, k}}}\right),
$$

where $\varsigma_{k-s}=\hat{x}_{k-s}^{-}$for $s=0$ and $\varsigma_{k-s}=\hat{x}_{k-s}$ for $s=1,2, \ldots, \ell . \Phi(\cdot)$ is the cumulative distribution function (CDF) of the random variable "." obeying the standard normal distribution.

The objectives of this paper are to i) design an optimal protocol-based TKF for system (1)-(6) in the LMMSE sense under the RRP; and ii) analyze the performance of the obtained filter via the evaluation index $\mathbb{E}\left\{P_{\tilde{x}_{k}}^{-}\right\}$.

\section{Protocol-BASEd TKF with Integral Measurements and Probabilistic Sensor Failures}

In this section, we aim to formalize an ameliorated Tobit Kalman filtering paradigm to surmount the challenges brought by the coexistence of sensor failures, integral observations and measurement censoring under the RRP. The formulation procedure differentiates itself from [2] in the following aspects: 1) a protocol-based Tobit regression model where impacts from the integral measurements, sensor failures and RRP are taken into consideration; and 2) extra computations of the gain and covariance matrices (resulting from the integral measurements and sensor failures) which comprise the augmentation of states, derivation with respect to the augmented state as well as calculations in terms of failure coefficients.

As integral measurements are often caused by the delayed sample collection and signal processing, the augmentation technique is first applied to system (1)-(6) to accommodate such integral effects. Letting

$$
\xi_{k} \triangleq\left[\begin{array}{llll}
x_{k}^{T} & x_{k-1}^{T} & \cdots & x_{k-\ell}^{T}
\end{array}\right]^{T},
$$

we have

$$
\begin{aligned}
\xi_{k} & =\mathcal{A}_{k} \xi_{k-1}+\mathcal{B}_{k} \omega_{k}, \\
y_{k} & =\gamma_{k} \sum_{m=1}^{p} \Gamma_{m, \hbar_{k}}\left(\Lambda_{m, k} \mathcal{C}_{m, k} \xi_{k}+v_{m, k}\right)+\left(1-\gamma_{k}\right) \tau,
\end{aligned}
$$


This article has been accepted for publication in a future issue of this journal, but has not been fully edited. Content may change prior to final publication Citation information: DOI10.1109/TSP.2020.3048245, IEEE Transactions on Signal Processing

where

$$
\begin{aligned}
\mathcal{A}_{k} & =\left[\begin{array}{cccc}
A_{k} & 0 & \cdots & 0 \\
I & 0 & \cdots & 0 \\
\vdots & \ddots & \ddots & \vdots \\
0 & \cdots & I & 0
\end{array}\right], \mathcal{B}_{k}=\left[\begin{array}{c}
I \\
0 \\
\vdots \\
0
\end{array}\right], \\
\mathcal{C}_{m, k} & =\left[\begin{array}{llll}
C_{m, k} & C_{m, k} & \cdots & C_{m, k}
\end{array}\right] .
\end{aligned}
$$

By means of augmentation, system (1)-(6) with integral measurements is converted into the integral-free one in (9)(10) at the cost of extra computations pertinent to the augmented state.

Let

$$
\begin{aligned}
& \hat{\xi}_{k}^{-} \triangleq \mathbb{E}\left\{\xi_{k} \mid y_{1: k-1}, \gamma_{1: k-1}\right\}, \quad \tilde{\xi}_{k}^{-} \triangleq \xi_{k}-\hat{\xi}_{k}^{-}, \\
& \hat{\xi}_{k} \triangleq \mathbb{E}\left\{\xi_{k} \mid y_{1: k}, \gamma_{1: k}\right\}, \quad \tilde{\xi}_{k} \triangleq \xi_{k}-\hat{\xi}_{k}, \\
& P_{\tilde{\xi}_{k}^{-}} \triangleq \mathbb{E}\left\{\tilde{\xi}_{k}^{-}\left(\tilde{\xi}_{k}^{-}\right)^{T} \mid y_{1: k-1}, \gamma_{1: k-1}\right\} \\
& P_{\tilde{\xi}_{k}} \triangleq \mathbb{E}\left\{\tilde{\xi}_{k} \tilde{\xi}_{k}^{T} \mid y_{1: k}, \gamma_{1: k}\right\}, \quad \zeta_{k} \triangleq \sum_{m=1}^{p} \Gamma_{m, \hbar_{k}} \Lambda_{m, k} \mathcal{C}_{m, k} \xi_{k}, \\
& \mathcal{R}_{k} \triangleq \sum_{m=1}^{p} \Gamma_{m, \hbar_{k}}^{2} R_{m, k}, \quad \vartheta_{k} \triangleq \frac{\mathcal{I}-\zeta_{k}}{\mathcal{R}_{k}}
\end{aligned}
$$

Before embarking on the filter design, the protocol-based Tobit regression model entailing the integral measurements and sensor failures is first derived.

Lemma 1: The expectation and variance of $y_{k}$ conditional on the observation sequence $y_{1: k-1}$ and censoring sequence $\gamma_{1: k}$ are

$$
\begin{aligned}
\mathbb{E}\left\{y_{k} \mid y_{1: k-1}, \gamma_{1: k}\right\} & =\gamma_{k}\left[\zeta_{k}+\sqrt{\mathcal{R}_{k}} \lambda\left(\vartheta_{k}\right)\right]+\left(1-\gamma_{k}\right) \tau, \\
\operatorname{var}\left\{y_{k} \mid y_{1: k-1}, \gamma_{1: k}\right\} & =\mathcal{R}_{k}\left[1-\varphi\left(\vartheta_{k}\right)\right],
\end{aligned}
$$

where

$$
\begin{aligned}
\lambda\left(\vartheta_{k}\right) & =\frac{\phi\left(\vartheta_{k}\right)}{1-\Phi\left(\vartheta_{k}\right)}, \\
\varphi\left(\vartheta_{k}\right) & =\lambda\left(\vartheta_{k}\right)\left[\lambda\left(\vartheta_{k}\right)-\vartheta_{k}\right] .
\end{aligned}
$$

Here, $\phi\left(\vartheta_{k}\right)$ and $\Phi\left(\vartheta_{k}\right)$ are, respectively, the PDF and CDF of the Gaussian random variable $\vartheta_{k}$ of following structures:

$$
\begin{aligned}
\phi\left(\vartheta_{k}\right) & =\frac{1}{\sqrt{2 \pi}} e^{-\frac{\left(\tau-\zeta_{k}\right)^{2}}{2 \mathcal{R}_{k}}}, \\
\Phi\left(\vartheta_{k}\right) & =\int_{-\infty}^{\tau} \frac{1}{\sqrt{2 \pi \mathcal{R}_{k}}} e^{-\frac{\left(y_{k}-\zeta_{k}\right)^{2}}{2 \mathcal{R}_{k}}} d_{y_{k}} .
\end{aligned}
$$

Proof: See Appendix A.

The protocol-based Tobit regression model given by Lemma 1 embodies the expectation and variance of $y_{k}$ conditional on sequences $y_{1: k-1}$ and $\gamma_{1: k}$. In contrast with its counterpart in [2] which barely cares about the measurement censoring in case of unknown $\gamma_{1: k}$, two noteworthy distinctions of model (11)-(12) can be encapsulated. The first distinction is the substitution of the censoring probability $\bar{\gamma}_{k}$ by the true censoring variable $\gamma_{k}$ owing to the exact acknowledgement of $\gamma_{1: k}$. The second distinction is the replacement of $C_{k} x_{k}$ (the product of the original measurement matrix $C_{k}$ and state $x_{k}$ ) and $R_{m, k}$ (the original noise covariance), respectively, by $\zeta_{k}=\sum_{m=1}^{p} \Gamma_{m, \hbar_{k}} \Lambda_{m, k} \mathcal{C}_{m, k} \xi_{k}$ (the sum of $p$ products with respect to the update coefficient $\Gamma_{m, \hbar_{k}}$, failure coefficient $\Lambda_{m, k}$, augmented measurement matrix $\mathcal{C}_{m, k}$ and augmented state $\xi_{k}$ ) and $\mathcal{R}_{k}=\sum_{m=1}^{p} \Gamma_{m, \hbar_{k}}^{2} R_{m, k}$ (the sum of $p$ products with regard to the update coefficient $\Gamma_{m, \hbar_{k}}$ and original noise covariance $R_{m, k}$ ) due to the involvement of the RRP, integral measurements and sensor failures. If these phenomena are disregarded, (11) and (12) would degrade to (8) and (11) in [2], respectively.

Remark 3: Thanks to (11)-(16), the Tobit regression model in [2] (without the acknowledgement of the measurement censoring) has been modified to the protocol-based one with integral measurements, sensor failures and acknowledged measurement censoring. Be aware that such a modification brings on board 1) the substitution of $\bar{\gamma}_{k}$ by $\gamma_{k}, C_{k} x_{k}$ by $\zeta_{k}$ and $R_{m, k}$ by $\mathcal{R}_{k}$ throughout all measurement-related terms; and 2) the emergence of a suite of new terms that further sophisticate the subsequent algorithm design.

Denote

$$
\begin{aligned}
& \mathfrak{C}_{m, k} \triangleq \Gamma_{m, \hbar_{k}} \bar{\Lambda}_{m, k} \mathcal{C}_{m, k}, \quad \hat{\zeta}_{k}^{-} \triangleq \sum_{m=1}^{p} \mathfrak{C}_{m, k} \hat{\xi}_{k}^{-}, \\
& \bar{\vartheta}_{k} \triangleq \frac{\tau-\hat{\zeta}_{k}^{-}}{\mathcal{R}_{k}}, \tilde{\Lambda}_{m, k} \triangleq \bar{\Lambda}_{m, k}^{2}+\breve{\Lambda}_{m, k}
\end{aligned}
$$

The following theorem presents the optimal protocol-based TKF in the LMMSE sense subject to integral measurements and sensor failures.

Theorem 1: The optimal protocol-based TKF for the augmented system (9)-(10) is

$$
\begin{aligned}
\hat{\xi}_{k}^{-} & =\mathcal{A}_{k} \hat{\xi}_{k-1}, \\
P_{\tilde{\xi}_{k}^{-}} & =\mathcal{A}_{k-1} P_{\tilde{\xi}_{k-1}} \mathcal{A}_{k-1}^{T}+\mathcal{B}_{k-1} Q_{k-1} \mathcal{B}_{k-1}^{T}, \\
\hat{\xi}_{k} & =\hat{\xi}_{k}^{-}+K_{k}\left(y_{k}-\hat{y}_{k}^{-}\right), \\
P_{\tilde{\xi}_{k}} & =P_{\tilde{\xi}_{k}^{-}}-K_{k} P_{\tilde{\xi}_{k}^{-} \tilde{y}_{k}^{-}}^{T} .
\end{aligned}
$$

The one-step measurement prediction and filtering gain are

$$
\begin{aligned}
\hat{y}_{k}^{-} & =\gamma_{k}\left[\hat{\zeta}_{k}^{-}+\sqrt{\mathcal{R}_{k}} \lambda\left(\bar{\vartheta}_{k}\right)\right]+\left(1-\gamma_{k}\right) \tau, \\
K_{k} & =P_{\tilde{\xi}_{k}^{-} \tilde{y}_{k}^{-}} P_{\tilde{y}_{k}^{-}}^{-1},
\end{aligned}
$$

where

$$
\begin{aligned}
P_{\tilde{\xi}_{k}^{-} \tilde{y}_{k}^{-}}= & P_{\tilde{\xi}_{k}^{-}}\left(\gamma_{k} \sum_{m=1}^{p} \mathfrak{C}_{m, k}\right)^{T} \\
P_{\tilde{y}_{k}^{-}}= & \gamma_{k} \sum_{m=1}^{p} \sum_{n=1}^{p} \mathfrak{C}_{m, k} P_{\tilde{\xi}_{k}^{-}}\left(\gamma_{k} \mathfrak{C}_{n, k}\right)^{T} \\
& +\gamma_{k} \sum_{m=1}^{p} \sum_{n=1}^{p} \Gamma_{m, \hbar_{k}} \tilde{\Lambda}_{m, k} \mathcal{C}_{m, k} P_{\xi_{k}}\left(\gamma_{k} \Gamma_{n, \hbar_{k}} \mathcal{C}_{n, k}\right)^{T} \\
& +\mathcal{R}_{k}\left[1-\varphi\left(\bar{\vartheta}_{k}\right)\right]
\end{aligned}
$$

Here, $\lambda\left(\bar{\vartheta}_{\hbar_{k}, k}\right)$ and $\varphi\left(\bar{\vartheta}_{\hbar_{k}, k}\right)$ can be calculated via (13)(14) by replacing $\zeta_{k}$ with $\hat{\zeta}_{k}^{-}$; and $P_{\xi_{k}}=\mathcal{A}_{k-1} P_{\xi_{k-1}} \mathcal{A}_{k-1}^{T}+$ $\mathcal{B}_{k-1} Q_{k-1} \mathcal{B}_{k-1}^{T}$.

Proof: See Appendix B. 
TABLE I: The Pseudocode of the Protocol-Based TKF

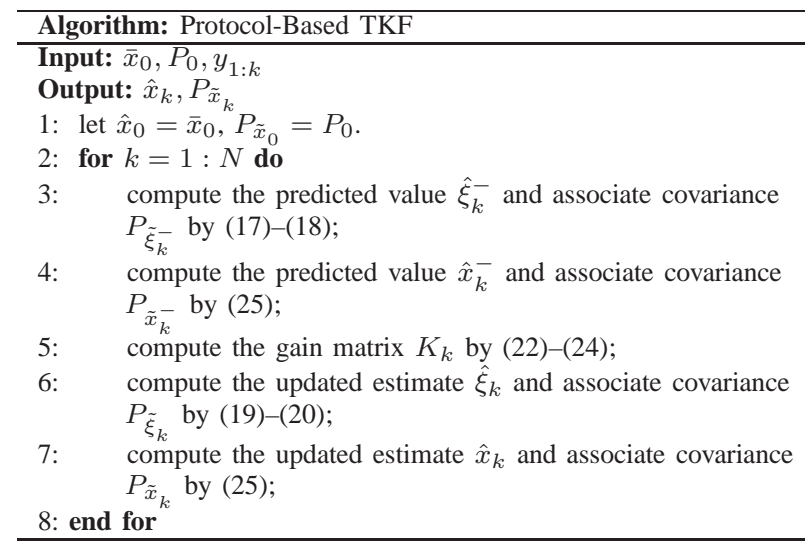

Two exceptional features can be spotted when comparing the proposed protocol-based TKF in Theorem 1 with its counterpart in [2]. One is the substitution of the term $\bar{\gamma}_{k} C_{k}$ (which is the product of the censoring probability and the original measurement coefficient) by the term $\gamma_{k} \sum_{m=1}^{p} \mathfrak{C}_{m, k}$ (which is the sum of $p$ products in relation to the known censoring variable $\gamma_{k}$ and the equivalent measurement coefficient $\mathfrak{C}_{m, k}$ ) in all prediction-related equations. The other is the emergence of the term $\gamma_{k} \sum_{m=1}^{p} \sum_{n=1}^{p} \Gamma_{m, \hbar_{k}} \tilde{\Lambda}_{m, k} \mathcal{C}_{m, k} P_{\xi_{k}}\left(\gamma_{k} \Gamma_{n, \hbar_{k}} \mathcal{C}_{n, k}\right)^{T}$ in the calculation of $P_{\tilde{y}_{k}^{-}}$. The first feature originates from the concurrence of the RRP, sensor failures, integral measurements and exact acknowledgement of the censoring phenomenon, whilst the second feature stems from the presence of sensor failures.

Let $\Pi \triangleq\left[\begin{array}{llll}I & 0 & \cdots & 0\end{array}\right]$. On the basis of Theorem 1, the following theorem presents the optimal protocol-based TKF for the original system (1)-(7).

Theorem 2: The optimal protocol-based TKF for system (1)-(7) is

$$
\left\{\begin{aligned}
\hat{x}_{k}^{-} & =\Pi \hat{\xi}_{k}^{-}, \\
\hat{x}_{k} & =\Pi \hat{\xi}_{k}, \\
P_{\tilde{x}_{k}^{-}} & =\Pi P_{\tilde{\xi}_{k}^{-}} \Pi^{T}, \\
P_{\tilde{x}_{k}} & =\Pi P_{\tilde{\xi}_{k}} \Pi^{T} .
\end{aligned}\right.
$$

Proof: Theorem 2 follows readily from Theorem 1 by noting the correlation between system (1)-(7) and system (9)(10).

Theorems 1-2, together with Lemma 1, constitute the protocol-based Tobit Kalman filtering algorithm with its pseudocode outlined in Table I.

It can be seen from Lemma 1 and Theorems 1-2 that, when implementing the presented protocol-based Tobit Kalman filtering algorithm with integral measurements and probabilistic sensor failures, the computational complexity mainly involves the matrix multiplication and inversion. In the matrix multiplication, the multiplication of matrices with dimensions $n_{x}(\ell+1) \times n_{x}(\ell+1)$ needs to be calculated, where $n_{x}$ is the dimension of the system state and $\ell$ is the time length required for the data collection. As to the matrix inversion, the inverse of matrices with dimensions $n_{y} \times n_{y}$ needs to be calculated, where $n_{y}$ is the dimension of the measurement $y_{k}$. Hence, the proposed filtering algorithm has the computational complexity of $O\left[\left(n_{x}(\ell+1)\right)^{3}+\left(n_{y}\right)^{3}\right]$.

Remark 4: The specifically tailored Tobit Kalman filtering architecture is composed of Lemma 1 and Theorems 1-2, and exhibits two extraordinary advantages. On one hand, system (1)-(7) under investigation is comprehensive for its inclusion of the RRP and multiple measurement uncertainties (e.g. measurement censoring, integral measurements and sensor failures) which are prevalently confronted in a myriad of application ranges and are elegantly settled in a holistic yet valid framework. On the other hand, in comparison with the filter in [2], the protocol-induced measurement update coefficient $\Gamma_{m, \hbar_{k}}$ and a bank of new terms arise in the development of our TKF, which transparently reveals the impacts from the RRP and measurement uncertainties. Explicitly, the term $\gamma_{k}$ throughout the paper manifests the influence of measurement censoring, the term $\mathcal{R}_{k}$ in Lemma 1 and Theorem 1 reveals the impact of the RRP, the term $\Pi$ in Theorem 2 reflects the effect of the integral measurements, the term $\gamma_{k} \sum_{m=1}^{p} \sum_{n=1}^{p} \Gamma_{m, \hbar_{k}} \tilde{\Lambda}_{m, k} \mathcal{C}_{m, k} P_{\xi_{k}}\left(\gamma_{k} \Gamma_{n, \hbar_{k}} \mathcal{C}_{n, k}\right)^{T}$ in Theorem 1 sketches the influence of sensor failures, and terms $\zeta_{k}$ and $\hat{\zeta}_{k}^{-}$characterize the simultaneous influence of the RRP, integral measurements and sensor failures.

It should also be noted that, the protocol-based Tobit Kalman filtering paradigm proposed in Theorems $1-2$ is stochastic due to its dependence on the random censoring variable $\gamma_{k}$. This indicates that the state estimates and associate error covariances are now functions of $\gamma_{k}$. Given such a stochastic filtering paradigm, a viable way to analyze its performance is to investigate the statistical property of the filtering error covariance $P_{\tilde{x}_{k}^{-}}$as shown in the later section.

\section{Performance Analysis}

In this section, we aim to come up with a holistic analysis of the filtering performance in relation to the proposed optimal protocol-based TKF. The analysis is performed by taking advantage of the mean estimation error covariance $\mathbb{E}\left\{P_{\tilde{x}_{k}^{-}}\right\}$. Considering the time-varying nature of the filter, we show that there exist self-propagating lower and upper bounds on $\mathbb{E}\left\{P_{\tilde{x}_{k}^{-}}\right\}$. The pursuit of such bounds complies with two principles: 1) the optimality of the filter motivates us to construct a suboptimal filter whose mean estimation error covariance is envisioned to be the upper bound on $\mathbb{E}\left\{P_{\tilde{x}_{k}^{-}}\right\}$; 2) the semi-positive definiteness of $P_{\tilde{\xi}_{k}^{-}}, P_{\xi_{k}}, \tilde{\Lambda}_{m, k}$ and $\mathcal{R}_{k}$ paves the way for us to envisage the lower bound on $\mathbb{E}\left\{P_{\tilde{x}_{k}^{-}}\right\}$ via some subtle matrix manipulations.

For the sake of notation brevity, we define $M_{k} \triangleq \mathbb{E}\left\{P_{\tilde{\xi}_{k}^{-}}\right\}$, $N_{k} \triangleq \mathbb{E}\left\{P_{\tilde{x}_{k}^{-}}\right\}$and $\bar{\varphi}_{k} \triangleq \varphi\left(\bar{\vartheta}_{k}\right)$. To begin with, the error covariance $P_{\tilde{\xi}_{k+1}^{-}}$in (17) is rearranged into

$$
P_{\tilde{\xi}_{k+1}^{-}}=\mathcal{A}_{k} P_{\tilde{\xi}_{k}^{-}} \mathcal{A}_{k}^{T}+\mathcal{B}_{k} Q_{k} \mathcal{B}_{k}^{T}-\mathcal{A}_{k} P_{\tilde{\xi}_{k}^{-}}\left(\gamma_{k} \sum_{s=1}^{p} \mathfrak{C}_{s, k}\right)^{T}
$$




$$
\begin{aligned}
& \times\left\{\gamma_{k} \sum_{m=1}^{p} \sum_{n=1}^{p} \mathfrak{C}_{m, k} P_{\tilde{\xi}_{k}^{-}}\left(\gamma_{k} \mathfrak{C}_{n, k}\right)^{T}\right. \\
& +\gamma_{k} \sum_{m=1}^{p} \sum_{n=1}^{p} \Gamma_{m, k} \tilde{\Lambda}_{m, k} \mathcal{C}_{m, k} P_{\xi_{k}}\left(\gamma_{k} \Gamma_{n, \hbar_{k}} \mathcal{C}_{n, k}\right)^{T} \\
& \left.+\mathcal{R}_{k}\left[1-\bar{\varphi}_{k}\right]\right\}^{-1} \gamma_{k} \sum_{t=1}^{p} \mathfrak{C}_{t, k} P_{\tilde{\xi}_{k}^{-}} \mathcal{A}_{k}^{T}
\end{aligned}
$$

Taking expectation on both sides of (26) leads to

$$
\begin{aligned}
M_{k+1}= & \mathcal{A}_{k} M_{k} \mathcal{A}_{k}^{T}+\mathcal{B}_{k} Q_{k} \mathcal{B}_{k}^{T} \\
& -\mathcal{A}_{k} \mathbb{E}\left\{P_{\tilde{\xi}_{k}^{-}}\left(\gamma_{k} \sum_{s=1}^{p} \mathfrak{C}_{s, k}\right)^{T}\right. \\
& \times\left[\gamma_{k} \sum_{m=1}^{p} \sum_{n=1}^{p} \mathfrak{C}_{m, k} P_{\tilde{\xi}_{k}^{-}}\left(\gamma_{k} \mathfrak{C}_{n, k}\right)^{T}\right. \\
& +\gamma_{k} \sum_{m=1}^{p} \sum_{n=1}^{p} \Gamma_{m, \hbar_{k}} \tilde{\Lambda}_{m, k} \mathcal{C}_{m, k} P_{\xi_{k}}\left(\gamma_{k} \Gamma_{n, \hbar_{k}} \mathcal{C}_{n, k}\right)^{T} \\
& \left.\left.+\mathcal{R}_{k}\left[1-\bar{\varphi}_{k}\right]\right]^{-1} \gamma_{k} \sum_{t=1}^{p} \mathfrak{C}_{t, k} P_{\tilde{\xi}_{k}^{-}}\right\} \mathcal{A}_{k}^{T} .
\end{aligned}
$$

It is apparent that the complex structure of the third term on the right side of (27) prevents the self-propagation of $M_{k+1}$, that is to say, the acknowledgement of $M_{k}$ is not sufficient for the determination of $M_{k+1}$, which further hinders us from finding the self-propagating bound on $M_{k}$. Nonetheless, it will be shown later that such an upper bound is well-expected through some subtle matrix manipulations.

Recalling that the optimal protocol-based TKF derived in Theorem 2 has a random filtering gain $K_{k}$, the following theorem is dedicated to the derivation of a self-propagating upper bound on $M_{k}$ via constructing a suboptimal protocolbased TKF with a deterministic filtering gain $K_{k}^{u}$.

Theorem 3: Let the initial condition $M_{0}^{u}>0$ be given. Calculate the matrix sequence $\left\{M_{k+1}^{u}\right\}_{k \geq 0}$ according to the following difference equation:

$$
\begin{aligned}
M_{k+1}^{u}= & \mathcal{A}_{k} M_{k}^{u} \mathcal{A}_{k}^{T}+\mathcal{B}_{k} Q_{k} \mathcal{B}_{k}^{T}-\mathcal{A}_{k} M_{k}^{u}\left(\bar{\gamma}_{k}^{u} \sum_{s=1}^{p} \mathfrak{C}_{s, k}\right)^{T} \\
& \times\left\{\bar{\gamma}_{k}^{u} \sum_{m=1}^{p} \sum_{n=1}^{p} \mathfrak{C}_{m, k} M_{k}^{u}\left(\bar{\gamma}_{k}^{u} \mathfrak{C}_{n, k}\right)^{T}\right. \\
& +\bar{\gamma}_{k}^{u} \sum_{m=1}^{p} \sum_{n=1}^{p} \Gamma_{m, \hbar_{k}} \tilde{\Lambda}_{m, k} \mathcal{C}_{m, k} P_{\xi_{k}}\left(\bar{\gamma}_{k}^{u} \Gamma_{n, k} \mathcal{C}_{n, k}\right)^{T} \\
& \left.+\mathcal{R}_{k}\left[1-\bar{\varphi}_{k}^{u}\right]\right\}^{-1} \bar{\gamma}_{k}^{u} \sum_{t=1}^{p} \mathfrak{C}_{t, k} M_{k}^{u} \mathcal{A}_{k}^{T} .
\end{aligned}
$$

Then, the calculated matrix $M_{k+1}^{u}$ satisfies

$$
M_{k+1} \leq M_{k+1}^{u},
$$

i.e. $M_{k+1}^{u}$ is a self-propagating upper bound on $M_{k+1}$, where $\bar{\gamma}_{k}^{u}$ and $\bar{\varphi}_{k}^{u}$ are the suboptimal counterparts of $\bar{\gamma}_{k}$ and $\bar{\varphi}_{k}$, respectively.

Proof: See Appendix C.
On account of the randomness of the gain matrix $K_{k}$ in Theorem 1, a straightforward idea is to construct a suboptimal protocol-based TKF by setting the gain matrix to be deterministic. Consequently, Theorem 3 presents an upper bound $M_{k}^{u}$ on $M_{k}$ and the suboptimal gain $K_{k}^{u}$ is obtained via minimizing the trace of $M_{k}^{u}$. Provided the initial condition, such an upper bound holds for all $k \geq 0$ due to the fact that, the filtering performance of the optimal protocol-based TKF should be no less than any of its suboptimal counterparts. It needs to be pointed out that, the self-propagation of $M_{k}^{u}$ holds regardless of the fact that $M_{k}$ is not self-propagating. This provides a feasible way to the online recursive computation of $M_{k}^{u}$. In addition, it can be observed from (28) that, the calculation of $M_{k}^{u}$ has close relationships with the augmented coefficients $\mathcal{A}_{k}$ and $\mathfrak{C}_{m, \hbar_{k}}$, updated coefficient $\Gamma_{m, k}$, failure variance $\tilde{\Lambda}_{m, k}$ and censoring probability $\bar{\gamma}_{k}^{u}$, which explicitly elucidates the impacts from the integral measurements, RRP, sensor failures and measurement censoring on.

Theorem 4: Let the initial condition $M_{0}^{l}>0$ be given. Calculate the matrix sequence $\left\{M_{k+1}^{l}\right\}_{k>0}$ according to the following difference equation:

$$
M_{k+1}^{l}=\left(1-\bar{\gamma}_{k}\right) \mathcal{A}_{k} M_{k}^{l} \mathcal{A}_{k}^{T}+\mathcal{B}_{k} Q_{k} \mathcal{B}_{k}^{T},
$$

Then, the calculated matrix $M_{k+1}^{l}$ satisfies

$$
M_{k+1}^{l} \leq M_{k+1}
$$

i.e. $M_{k+1}^{l}$ is a self-propagating lower bound on $M_{k+1}$.

Proof: See Appendix D.

Making full use of some subtle matrix manipulations, a selfpropagating lower bound $M_{k}^{l}$ on $M_{k}$ is acquired in Theorem 4 based on the semi-positive definiteness of $P_{\tilde{\xi}_{k}^{-}}, P_{\xi_{k}}, \tilde{\Lambda}_{m, k}$ and $\mathcal{R}_{k}$. Casting insights into Theorem 4 , a remarkable finding is that, the calculation of $M_{k}^{l}$ tightly hinges on the augmented coefficients $\mathcal{A}_{k}$ and $\mathcal{B}_{k}$ and censoring probability $\bar{\gamma}_{k}$, which noticeably characterizes the effects of the RRP, integral measurements, sensor failures and measurement censoring.

Keeping in mind the relationship between $P_{\tilde{\xi}_{k}^{-}}$and $P_{\tilde{x}_{k}^{-}}$as shown in Theorem 2, we arrive at the following theorem.

Theorem 5: There exist an upper bound $N_{k}^{u}$ and a lower bound $N_{k}^{l}$ on the mean error covariance $N_{k}$ such that

$$
N_{k}^{l} \leq N_{k} \leq N_{k}^{u}
$$

holds for all $k \geq 0$, where $N_{k}^{u}=\Pi M_{k}^{u} \Pi^{T}$ and $N_{k}^{l}=$ $\Pi M_{k}^{l} \Pi^{T}$.

Proof: The result follows noticeably from Theorems 2-4.

Remark 5: Theorems 5 manifests the upper and lower bounds on the mean estimation error covariance $N_{k}$ under the RRP and multiple measurement uncertainties (e.g. measurement censoring, integral measurements and sensor failures). The concurrence of the RRP and uncertainties provokes substantial difficulties in the assessment of the filtering performance which can be encapsulated from the following two aspects. 1) The exact acknowledgement of the censoring phenomenon gives rise to a time-varying and stochastic protocolbased TKF as shown in Theorems 1-2, which prevents us from carrying out a rigorous convergence analysis on the proposed 
filter. As such, we turn to explore the self-propagating upper and lower bounds on the mean error covariance $N_{k}$. The upper bound is achieved by establishing a suboptimal protocol-based TKF whose gain matrix is independent of $\gamma_{k}$, and the lower bound is acquired by resorting to subtle matrix manipulations based on the semi-positive definiteness of matrices $P_{\tilde{\xi}_{k}^{-}}, P_{\xi_{k}}$, $\tilde{\Lambda}_{m, k}$ and $\mathcal{R}_{k}$. 2) The conjunction of the RRP and measurement uncertainties gives rise to a set of protocol-induced and uncertainty-induced terms, significantly sophisticating the design of the suboptimal filter and the implementation of the involved matrix manipulations. To sum up, the simultaneous $\mathrm{p}$ resence of the RRP and measurement uncertainties ineluctably results in distinctive filter design techniques and performance analysis procedures which are elegantly orchestrated through Theorems 1-5.

\section{ILlustrative EXAMPLE}

In this section, we leverage an oscillator example (modified from [2]) to elucidate the applicability of the presented filter design strategy and performance analysis mechanism.

Denote the root mean-squared errors (RMSEs) of $x_{k}^{1}$ and $x_{k}^{2}$, respectively, as $\mathrm{RMSE} 1 \triangleq \sqrt{\frac{1}{M} \sum_{i=1}^{M}\left(x_{k}^{1(i)}-\hat{x}_{k}^{1(i)}\right)^{2}}$ and $\mathrm{RMSE} 2 \triangleq \sqrt{\frac{1}{M} \sum_{i=1}^{M}\left(x_{k}^{2(i)}-\hat{x}_{k}^{2(i)}\right)^{2}}$, and the mean error covariance trace (MECT) as MECT $\triangleq(1 / M) \sum_{i=1}^{M} \operatorname{tr}\left(P_{\tilde{x}_{k}^{-}}^{(i)}\right)$ where $M$ is the number of Monte Carlo trials.

Let the discrete time-varying system (1)-(6) have following parameters:

$$
\begin{aligned}
A_{k} & =\left[\begin{array}{cc}
\cos (w) & -\sin (w) \\
\sin (w) & \cos (w)
\end{array}\right], w=0.052 \pi, \\
R_{1, k} & =R_{2, k}=1, \tau=0, p=2, \ell=2, \\
C_{1, k} & =\left[\begin{array}{ll}
1 & 0
\end{array}\right], C_{2, k}=\left[\begin{array}{ll}
0 & 1
\end{array}\right], \bar{x}_{0}=\left[\begin{array}{ll}
5 & 0
\end{array}\right]^{T}, \\
Q_{k} & =\operatorname{diag}\{0.0025,0.0025\}, P_{0}=I_{2}, M_{0}=I_{6},
\end{aligned}
$$

where $\ell$ is the interval required for the measurement integral. The oscillator example concerns about the estimation of ballistic roll rates in case of the noisy dynamic model and uncertain magnetometer data. Sensor failure rates $\Lambda_{m, k}(m=1,2)$ can be determined via statistical tests, and are supposed to be regulated by the PDF $p(s)=0.05 \delta(s)+0.10 \delta(s-0.5)+$ $0.85 \delta(s-1)$. Apparently, expectations and variances of $\Lambda_{m, k}$ are computed as $\bar{\Lambda}_{m, k}=0.90$ and $\tilde{\Lambda}_{m, k}=0.065$. Note that the dynamic model is corrupted by ambient disturbances entering the system via $\omega_{k}$. The magnetometer sampling is subject to sensor failures and integral measurements, and the data transmission is scheduled by the RRP.

Fig. 2 depicts the true state values and associate estimates generated by the protocol-based Tobit Kalman filter (which is named as PBTKF and is capable of tackling censored observations under the RRP) and the protocol-based Tobit Kalman filter with integral measurements and sensor failures (which is named as PBTKF-IMSF and is capable of simultaneously tackling integral measurements, sensor failures and censored observations under the RRP). Fig. 3 plots the comparison result in RMSE between the PBTKF and PBTKF-IMSF after
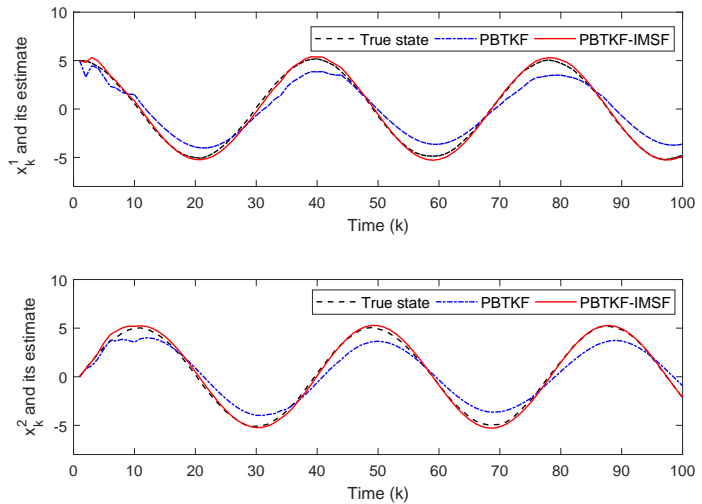

Fig. 2: True values of the first and second dimensions of the state and their estimates.
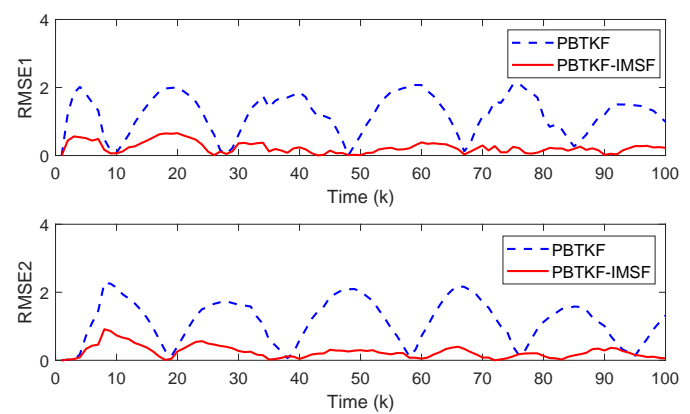

Fig. 3: Performance comparison in RMSE1 and RMSE2.

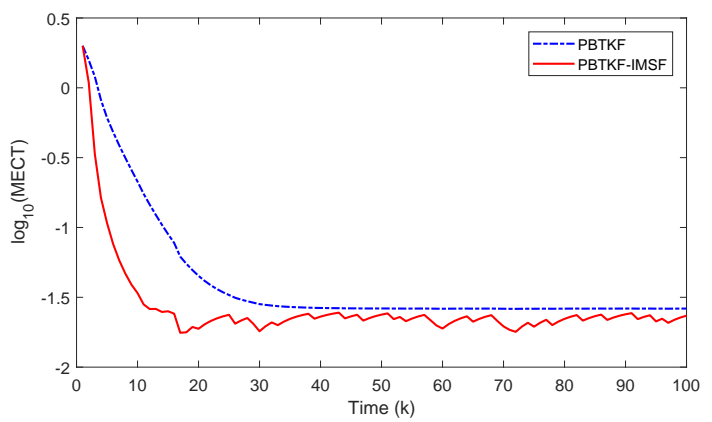

Fig. 4: Performance comparison in $\log _{10}$ (MECT).

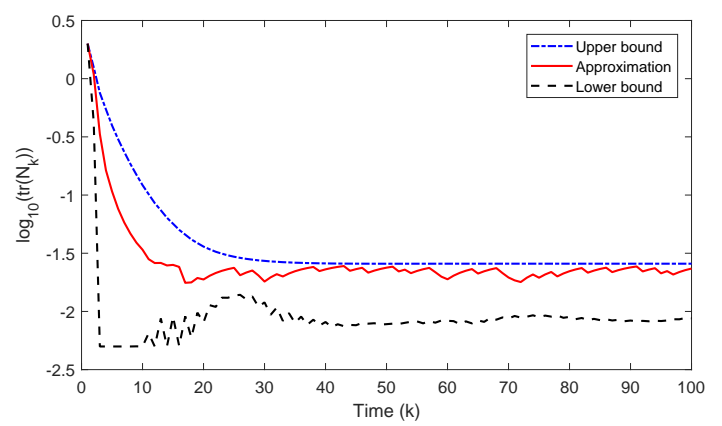

Fig. 5: Comparison among $\log _{10}\left(\operatorname{tr}\left\{N_{k}\right\}\right), \log _{10}\left(\operatorname{tr}\left\{N_{k}^{u}\right\}\right)$ and $\log _{10}\left(\operatorname{tr}\left\{N_{k}^{l}\right\}\right)$. 

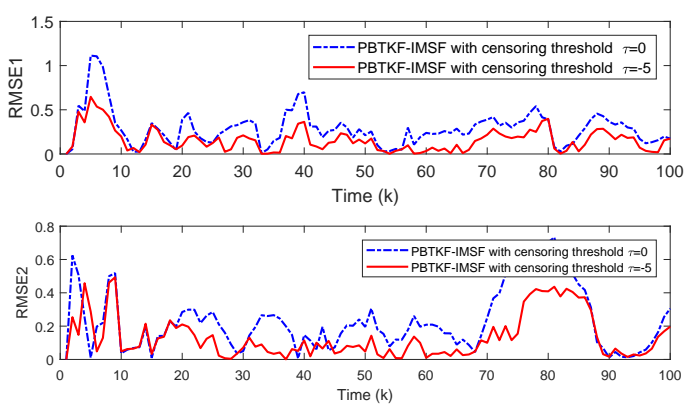

Fig. 6: RMSE1 and RMSE2 comparison of the PBTKF-IMSFs with different censoring thresholds: $\tau=-5$ and $\tau=0$.
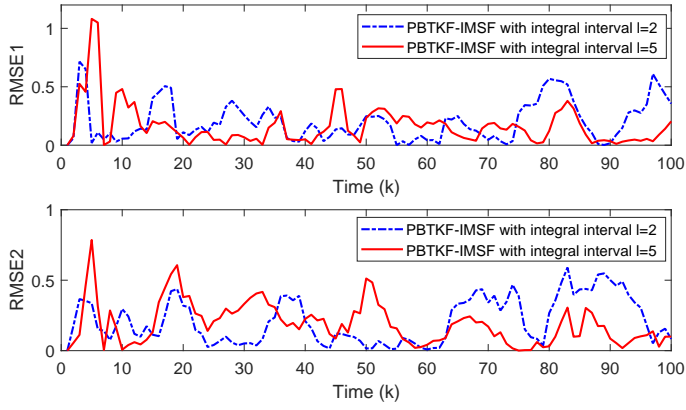

Fig. 7: RMSE1 and RMSE2 comparison of the PBTKF-IMSFs with different integral intervals: $\ell=2$ and $\ell=5$.

1000 independent Mote Carlo trials. It is witnessed from Fig. 2 that, the PBTKF-IMSF manages to track the true state values precisely, whilst the PBTKF appears to have considerable deviations from the true state values. Besides, it is sketched in Fig. 3 that, the RMSE curve of the PBTKF-IMSF resides lower than that of the PBTKF, indicating that issues of integral measurements and sensor failures are suitably addressed in the PBTKF-IMSF, whilst they are not settled in the PBTKF.

Next, letting $A_{k}=0.9\left[\begin{array}{cc}\cos (w) & -\sin (w) \\ \sin (w) & \cos (w)\end{array}\right]$ and $M=$ 1000 , the comparison result between the PBTKF and PBTKFIMSF in MECT is manifested in Fig. 4. Besides, relationships among the trace of the mean error covariance $N_{k}$, its upper bound $N_{k}^{u}$ and lower bound $N_{k}^{l}$ (calculated by our PBTKFIMSF) are sketched in Fig. 5. Due to the impossibility of analytically computing $N_{k}, \log _{10}\left(\operatorname{tr}\left\{N_{k}\right\}\right)$ is approximated by $\log _{10}(\mathrm{MECT})$. It can be spotted from Fig. 3 that, the value of $\log _{10}\left(\operatorname{tr}\left\{N_{k}\right\}\right)$ calculated by the PBTKF-IMSF is always smaller than that calculated by the PBTKF, certifying the superiority of our PBTKF-IMSF in simultaneously handling integral measurements and sensor failures over the PBTKF. Additionally, it is observed from Fig. 4 that, the curve of $\log _{10}\left(\operatorname{tr}\left\{N_{k}\right\}\right)$ always resides between those of $\log _{10}\left(\operatorname{tr}\left(N_{k}^{u}\right)\right)$ and $\log _{10}\left(\operatorname{tr}\left(N_{k}^{l}\right)\right)$, which justifies the statement in Theorem 5 that $N_{k}^{u}$ and $N_{k}^{l}$ can be, respectively, treated as reasonable upper and lower bounds on $N_{k}$.

In addition, to further demonstrate the filtering performance of our developed PBTKF-IMSF under different measurement
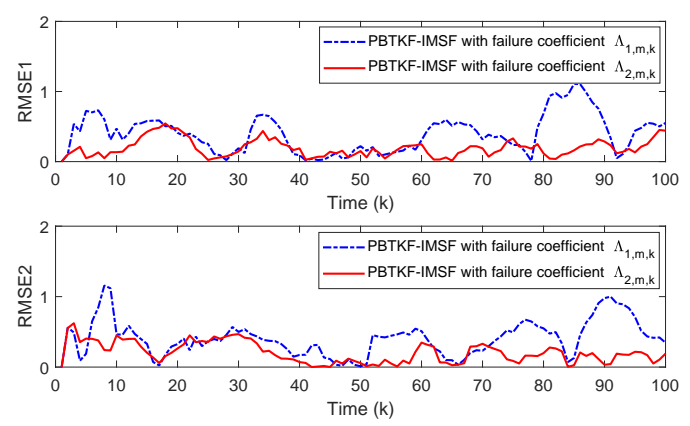

Fig. 8: RMSE1 and RMSE2 comparison of the PBTKF-IMSFs with different failure coefficients: $\Lambda_{1, m, k}$ and $\Lambda_{2, m, k}$.

censoring, integral measurements and sensor failures, simulation scenarios with different values of censoring thresholds $\tau$, integral intervals $\ell$ and failure coefficients $\Lambda_{m, k}$ are tested. Let us consider two sets of censoring thresholds $\{-5,0\}$, two sets of integral intervals $\{2,5\}$ and two sets of failure coefficients $\left\{\Lambda_{1, m, k}, \Lambda_{2, m, k}\right\}$ where $\Lambda_{1, m, k}$ and $\Lambda_{2, m, k}$ are, respectively, regulated by PDFs $p_{1}(s)=0.35 \delta(s)+0.10 \delta(s-$ $0.5)+0.55 \delta(s-1)$ and $p_{2}(s)=0.05 \delta(s)+0.10 \delta(s-0.5)+$ $0.85 \delta(s-1)$. Apparently, expectations and variances of $\Lambda_{1, m, k}$ and $\Lambda_{2, m, k}$ are computed as $\bar{\Lambda}_{1, m, k}=0.6, \tilde{\Lambda}_{1, m, k}=0.215$, $\bar{\Lambda}_{2, m, k}=0.9$ and $\tilde{\Lambda}_{2, m, k}=0.065$.

After 1000 independent Mote Carlo trials, RMSE results of our PBTKF-IMSF under different censoring thresholds, integral intervals and failure coefficients are, respectively, sketched in Figs. 6-8. It is witnessed from Fig. 6 that, the RMSE curve generated in case of $\tau=-5$ always locates lower than that generated in case of $\tau=0$. This is reasonable as a smaller censoring threshold $\tau$ indicates that, less measurements are inclined to the censoring phenomenon and more measurement information can be utilized in state estimation. This undoubtedly leads to better filtering accuracy of the PBTKF-IMSF.

One observes from Fig. 7 that, the RMSE curves generated in cases of $\ell=2$ and $\ell=5$ are intertwined with each other, indicating that no deterministic relationship exists between the length of the integral interval and the filtering accuracy of our PBTKF-IMSF. As a matter of fact, in case of the integral measurement, the sensor observation is actually proportional to the integral of system states within a prescribed time interval $\ell$, and hence the information from not only the current system state, but also the past system states, can be utilized for the estimation of the current system state. The variation of the integral interval $\ell$ (from $\ell=2$ to $\ell=5$ ) implies that more information on the past system states is introduced to the sensor observation. Nevertheless, the extra information about the past system states does not contain any knowledge about the current system state and makes no contribution to the estimation of the current system state. As a result, it can be concluded that the variation of the integral interval $\ell$ has no explicit influence on the filtering performance of our PBTKFIMSF, which coincides with the observation made from Fig. 7.

It can be spotted from Figs. 8 that, the RMSE curve gener- 
ated by our PBTKF-IMSF with the failure coefficient $\Lambda_{1, m, k}$ always locates lower than that generated by our PBTKFIMSF with the failure coefficient $\Lambda_{2, m, k}$. This is reasonable as it can be observed from the expectations and variances of $\Lambda_{1, m, k}$ and $\Lambda_{2, m, k}$ that, the sensor with the failure coefficient $\Lambda_{2, m, k}$ is more likely to have failures than the sensor with the failure coefficient $\Lambda_{1, m, k}$. This indicates that the sensor with the failure coefficient $\Lambda_{1, m, k}$ is capable of providing more state information to the filter than the sensor with the failure coefficient $\Lambda_{2, m, k}$. As a result, the PBTKF-IMSF with the failure coefficient $\Lambda_{1, m, k}$ outperforms the PBTKF-IMSF with the failure coefficient $\Lambda_{2, m, k}$ in filtering accuracy.

\section{CONCLUSION}

In this paper, we have settled the protocol-based Tobit Kalman filtering problem susceptible to phenomena of integral measurements and sensor failures. The integral measurements have been described as functions of states over a time period, the sensor failures have been characterized by random variables taking values on the interval $[0,1]$ according to certain PDFs with known means and variances, and the data transmission in the network has been commanded by the RRP. These phenomena have been elaborately addressed via bringing on board a couple of new terms, which have provoked additional calculations in measurement predictions, gain matrices as well as error covariances. Fortunately, the increased calculations are recursive or can be conducted off-line. Consequently, the devised filter is propitious for online scenarios. Further analysis has been performed to assess the filtering performance and a sufficient condition has been pinned down to guarantee the existence of self-propagating upper and lower bounds on the mean estimation error covariance. Finally, an application case study has been exploited to verify the efficacy of the developed method.

In addition, related topics for further research work can be listed as follows.

- Tobit Kalman filtering problems subject to multiple nonlinearities, e.g. the stochastic nonlinearity [19] and saturation nonlinearity [10], [38].

- Tobit Kalman filtering problems under different communication protocols, e.g. the event-triggered protocol [27] and try-once-discard protocol [41].

- Tobit Kalman filtering problems with various networkinduced phenomena, e.g. the signal quantization [20], [50] and channel fading [8].

\section{APPENDIX}

\section{A. Proof of Lemma 1}

Proof: It follows from (2)-(3) that $\bar{y}_{k}$ is a Gaussian variable with the mean $\zeta_{k}$ and variance $\mathcal{R}_{k}$. Then, the PDF of $y_{k}$ conditioned on $y_{1: k-1}$ and $\gamma_{1: k}$ can be expressed as

$$
\begin{aligned}
f\left(y_{k} \mid y_{1: k-1}, \gamma_{1: k}\right)= & \frac{1}{\sqrt{\mathcal{R}_{k}}} \phi\left(\frac{y_{k}-\zeta_{k}}{\sqrt{\mathcal{R}_{k}}}\right) u\left(y_{k}-\tau\right) \\
& +\delta\left(\tau-y_{k}\right) \Phi\left(\vartheta_{k}\right)
\end{aligned}
$$

where $\phi\left(\frac{y_{k}-\zeta_{k}}{\sqrt{\mathcal{R}_{k}}}\right)$ and $\Phi\left(\vartheta_{k}\right)$ are calculated via (15)-(16), and $u\left(y_{k}-\tau\right)$ is the unit step function. Taking advantage of (4), (33) is further translated into

$$
f\left(y_{k} \mid y_{1: k-1}, \gamma_{1: k}\right)=\frac{\gamma_{k}}{\sqrt{\mathcal{R}_{k}}} \frac{\phi\left(\frac{y_{k}-\zeta_{k}}{\sqrt{\mathcal{R}_{k}}}\right)}{1-\Phi\left(\vartheta_{k}\right)}+\left(1-\gamma_{k}\right) .
$$

In the light of (34), the conditional expectation of $y_{k}$ is

$$
\begin{aligned}
& \mathbb{E}\left\{y_{k} \mid y_{1: k-1}, \gamma_{1: k}\right\} \\
= & \int_{-\infty}^{+\infty} \eta_{k} f\left(\eta_{k} \mid \eta_{1: k-1}, \gamma_{1: k}\right) d_{\eta_{k}} \\
= & \gamma_{k} \int_{\tau}^{+\infty} \frac{\eta_{k}}{\sqrt{\mathcal{R}_{k}}} \frac{\phi\left(\frac{\eta_{k}-\zeta_{k}}{\sqrt{\mathcal{R}_{k}}}\right)}{1-\Phi\left(\vartheta_{k}\right)} d_{\eta_{k}}+\left(1-\gamma_{k}\right) \tau \\
= & \gamma_{k}\left[\zeta_{k}+\sqrt{\mathcal{R}_{k}} \lambda\left(\vartheta_{k}\right)\right]+\left(1-\gamma_{k}\right) \tau,
\end{aligned}
$$

which is exactly the same as (11), where $\lambda\left(\vartheta_{k}\right)$ is calculated via (13). In line with (11), we get

$$
\begin{aligned}
\mathbb{E}\left\{y_{k} \mid y_{1: k-1}, \gamma_{1: k-1}, \gamma_{k}\right. & =1\}=\zeta_{k}+\sqrt{\mathcal{R}_{k}} \lambda\left(\vartheta_{k}\right), \\
\mathbb{E}\left\{y_{k} \mid y_{1: k-1}, \gamma_{1: k-1}, \gamma_{k}\right. & =0\}=\tau, \\
\operatorname{var}\left\{y_{k} \mid y_{1: k-1}, \gamma_{1: k-1}, \gamma_{k}\right. & =0\}=0 .
\end{aligned}
$$

As a result, we have

$$
\begin{aligned}
\operatorname{var}\left\{y_{k} \mid y_{1: k-1}, \gamma_{1: k}\right\}= & \operatorname{var}\left\{y_{k} \mid y_{1: k-1}, \gamma_{1: k-1}, \gamma_{k}=1\right\} \\
= & \mathbb{E}\left\{y_{k}^{2} \mid y_{1: k-1}, \gamma_{1: k-1}, \gamma_{k}=1\right\} \\
& -\left(\mathbb{E}\left\{y_{k} \mid y_{1: k-1}, \gamma_{1: k-1}, \gamma_{k}=1\right\}\right)^{2} \\
= & \mathcal{R}_{k}\left[1-\varphi\left(\vartheta_{k}\right)\right],
\end{aligned}
$$

which is exactly the same as (12), where $\varphi\left(\vartheta_{k}\right)$ is calculated via (14). This completes the proof.

\section{B. Proof of Theorem 1}

Proof: A straightforward exploitation of the orthogonality projection principle [3] to system (9)-(10) yields (17)-(20) where the optimal gain matrix is computed by (22). The combination of (10) and (11) generates

$$
\begin{aligned}
\tilde{y}_{k}^{-}= & \gamma_{k} \sum_{m=1}^{p} \Gamma_{m, \hbar_{k}}\left(\Lambda_{m, k} \mathcal{C}_{m, k} \xi_{k}+v_{m, k}\right) \\
& -\gamma_{k}\left[\sum_{m=1}^{p} \Gamma_{m, \hbar_{k}} \bar{\Lambda}_{m, k} \mathcal{C}_{m, k} \hat{\xi}_{k}^{-}+\sqrt{\mathcal{R}_{k}} \lambda\left(\vartheta_{k}\right)\right] \\
= & \gamma_{k} \sum_{m=1}^{p} \Gamma_{m, \hbar_{k}}\left(\bar{\Lambda}_{m, k} \mathcal{C}_{m, k} \tilde{\xi}_{k}^{-}+\left(\Lambda_{m, k}-\bar{\Lambda}_{m, k}\right) \mathcal{C}_{m, k} \xi_{k}\right) \\
& +\gamma_{k}\left(\sum_{m=1}^{p} \Gamma_{m, \hbar_{k}} v_{m, k}-\sqrt{\mathcal{R}_{k}} \lambda\left(\vartheta_{k}\right)\right),
\end{aligned}
$$

where $\lambda\left(\vartheta_{k}\right)$ can be calculated via (13) by replacing $\zeta_{k}$ with $\hat{\zeta}_{k}^{-}$.

Putting (35) into the definitions of $P_{\tilde{\xi}_{k}^{-} \tilde{y}_{k}^{-}}$and $P_{\tilde{y}_{k}^{-}}$, respectively, gives (23) and

$$
P_{\tilde{y}_{k}^{-}}=\mathbb{E}\left\{\tilde{y}_{k}^{-}\left(\tilde{y}_{k}^{-}\right)^{T} \mid y_{1: k-1}, \gamma_{1: k}\right\}
$$




$$
\begin{aligned}
= & \gamma_{k} \sum_{m=1}^{p} \sum_{n=1}^{p} \mathfrak{C}_{m, k} P_{\tilde{\xi}_{k}^{-}}\left(\gamma_{k} \mathfrak{C}_{n, k}\right)^{T} \\
& +\gamma_{k} \sum_{m=1}^{p} \sum_{n=1}^{p} \Gamma_{m, \hbar_{k}} \tilde{\Lambda}_{m, k} \mathcal{C}_{m, k} P_{\xi_{k}}\left(\gamma_{k} \Gamma_{n, \hbar_{k}} \mathcal{C}_{n, k}\right)^{T} \\
& +\operatorname{var}\left\{y \hbar_{k}, k \mid y \hbar_{1: k-1}, \gamma_{\hbar_{1: k}}\right\} \\
= & \gamma_{k} \sum_{m=1}^{p} \sum_{n=1}^{p} \mathfrak{C}_{m, k} P_{\tilde{\xi}_{k}^{-}}\left(\gamma_{k} \mathfrak{C}_{n, k}\right)^{T} \\
& +\gamma_{k} \sum_{m=1}^{p} \sum_{n=1}^{p} \Gamma_{m, \hbar_{k}} \tilde{\Lambda}_{m, k} \mathcal{C}_{m, k} P_{\xi_{k}}\left(\gamma_{k} \Gamma_{n, \hbar_{k}} \mathcal{C}_{n, k}\right)^{T} \\
& +\mathcal{R}_{k}\left[1-\varphi\left(\bar{\vartheta}_{k}\right)\right],
\end{aligned}
$$

which is exactly the same as (24). This completes the proof.

\section{Proof of Theorem 3}

Proof: Now, let us concentrate on designing a suboptimal protocol-based TKF whose gain matrix $K_{k}^{u}$ does not hinge on $\gamma_{k}$. Let $\bar{\gamma}_{k}^{u}, \tilde{\xi}_{k}^{u-}, \tilde{y}_{k}^{u-}, \bar{\varphi}_{k}^{u}, P_{\tilde{\xi}_{k}}^{u}, P_{\tilde{\xi}_{k}^{-}}^{u}, K_{k}^{u}, P_{\tilde{\xi}_{k}^{-} \tilde{y}_{k}^{-}}^{u}, P_{\tilde{y}_{k}^{-}}^{u}$ and $M_{k}^{u}$, respectively, be the suboptimal counterparts of $\bar{\gamma}_{k}, \tilde{\xi}_{k}^{-}$, $\tilde{y}_{k}^{-}, \bar{\varphi}_{k}, P_{\tilde{\xi}_{k}}, P_{\tilde{\xi}_{k}^{-}}, K_{k}, P_{\tilde{\xi}_{k}^{-} \tilde{y}_{k}^{-}}, P_{\tilde{y}_{k}^{-}}$and $M_{k}$. In this respect, we have

$$
\begin{aligned}
P_{\tilde{\xi}_{k}}^{u}= & \mathbb{E}\left\{\left(\tilde{\xi}_{k}^{u-}-K_{k}^{u} \tilde{y}_{k}^{u-}\right)\left(\tilde{\xi}_{k}^{u-}-K_{k}^{u} \tilde{y}_{k}^{u-}\right)^{T} \mid y_{1: k}, \gamma_{1: k}\right\} \\
= & P_{\xi_{k}^{-}}^{u}-P_{\tilde{\xi}^{-}}^{u} \tilde{y}_{k}^{-} \\
& +K_{k}^{u} P_{\tilde{y}_{k}^{-}}^{u}\left(K_{k}^{u}\right)^{T}-K_{k}^{u}\left(P_{\tilde{\xi}_{k}^{-}}^{u} \tilde{y}_{k}^{-}\right)^{T} \\
P_{\tilde{\xi}_{k+1}^{-}}^{u}= & \mathbb{E}\left\{\left(\mathcal{A}_{k} \tilde{\xi}_{k}^{u-}+\mathcal{B}_{k} \omega_{k}-\mathcal{A}_{k} K_{k}^{u} \tilde{y}_{k}^{u-}\right)\right. \\
& \left.\times\left(\mathcal{A}_{k} \tilde{\xi}_{k}^{u-}+\mathcal{B}_{k} \omega_{k}-\mathcal{A}_{k} K_{k}^{u} \tilde{y}_{k}^{u-}\right)^{T} \mid y_{1: k}, \gamma_{1: k+1}\right\} \\
= & \mathcal{A}_{k} P_{\tilde{\xi}_{k}^{-}}^{u} \mathcal{A}_{k}^{T}+\mathcal{B}_{k} Q_{k} \mathcal{B}_{k}^{T}-\mathcal{A}_{k} P_{\tilde{\xi}_{k}^{-}}^{u} \tilde{y}_{k}^{-}\left(\mathcal{A}_{k} K_{k}^{u}\right)^{T} \\
& -\left[\mathcal{A}_{k} P_{\tilde{\xi}_{k}^{-}}^{u} \tilde{y}_{k}^{-}\left(\mathcal{A}_{k} K_{k}^{u}\right)^{T}\right]^{T}+\mathcal{A}_{k} K_{k}^{u} P_{\tilde{y}_{k}^{-}}^{u}\left(\mathcal{A}_{k} K_{k}^{u}\right)^{T} .
\end{aligned}
$$

Taking expectation on both sides of (36) yields

$$
\begin{aligned}
\mathbb{E}\left\{P_{\tilde{\xi}_{k}}^{u}\right\}= & \mathbb{E}\left\{P_{\tilde{\xi}_{k}^{-}}^{u}\right\}-\mathbb{E}\left\{P_{\tilde{\xi}_{k}^{-}}^{u} \tilde{y}_{k}^{-}\right\}\left(K_{k}^{u}\right)^{T}-K_{k}^{u} \\
& \times \mathbb{E}\left\{\left(P_{\tilde{\xi}_{k}^{-} \tilde{y}_{k}^{-}}^{u}\right)^{T}\right\}+K_{k}^{u} \mathbb{E}\left\{P_{\tilde{y}_{k}^{-}}^{u}\right\}\left(K_{k}^{u}\right)^{T} .
\end{aligned}
$$

In this regard, the suboptimal gain $K_{k}^{u}$ can be determined by minimizing the trace of $\mathbb{E}\left\{P_{\tilde{\xi}_{k}}^{u}\right\}$. Taking the matrix trace and derivative with respect to $K_{k}^{u}$ on both sides of (38), we have

$$
\begin{aligned}
\frac{\partial \operatorname{tr}\left\{\mathbb{E}\left\{P_{\tilde{\xi}_{k}}^{u}\right\}\right\}}{\partial K_{k}^{u}}= & -2 \operatorname{tr}\left\{\mathbb{E}\left\{P_{\tilde{\xi}_{k}^{-} \tilde{y}_{k}^{-}}^{u}\right\}\right\} \\
& +2 \operatorname{tr}\left\{K_{k}^{u} \mathbb{E}\left\{P_{\tilde{y}_{k}^{-}}^{u}\right\}\right\} .
\end{aligned}
$$

Letting (39) be equal to zero generates

$$
K_{k}^{u}=\mathbb{E}\left\{P_{\tilde{\xi}_{k}^{-} \tilde{y}_{k}^{-}}^{u}\right\} \mathbb{E}^{-1}\left\{P_{\tilde{y}_{k}^{-}}^{u}\right\} .
$$

Taking expectation on both sides of (37), we arrive at

$$
\begin{aligned}
M_{k+1}^{u}= & \mathcal{A}_{k} M_{k}^{u} \mathcal{A}_{k}^{T}+\mathcal{B}_{k} Q_{k} \mathcal{B}_{k}^{T}-\mathcal{A}_{k} \mathbb{E}\left\{P_{\tilde{\xi}_{k}^{-}}^{u} \tilde{y}_{k}^{-}\left(K_{k}^{u}\right)^{T}\right\} \mathcal{A}_{k}^{T} \\
& -\left[\mathcal{A}_{k} \mathbb{E}\left\{P_{\tilde{\xi}_{k}^{u}}^{u} \tilde{y}_{k}^{-}\left(K_{k}^{u}\right)^{T}\right\} \mathcal{A}_{k}^{T}\right]^{T} \\
& +\mathcal{A}_{k} \mathbb{E}\left\{K_{k}^{u} P_{\tilde{\xi}_{k}^{-} \tilde{y}_{k}^{-}}^{u}\left(K_{k}^{u}\right)^{T}\right\} \mathcal{A}_{k}^{T} .
\end{aligned}
$$

Bearing in mind the non-randomness of $K_{k}^{u}$ and substituting (40) into (41), we have

$$
\begin{aligned}
M_{k+1}^{u}= & \mathcal{A}_{k} M_{k}^{u} \mathcal{A}_{k}^{T}+\mathcal{B}_{k} Q_{k} \mathcal{B}_{k}^{T}-\mathcal{A}_{k} K_{k}^{u} \mathbb{E}^{T}\left\{P_{\tilde{\xi}_{k}^{u} \tilde{y}_{k}^{-}}^{u}\right\} \mathcal{A}_{k}^{T} \\
= & \mathcal{A}_{k} M_{k}^{u} \mathcal{A}_{k}^{T}+\mathcal{B}_{k} Q_{k} \mathcal{B}_{k}^{T}-\mathcal{A}_{k} M_{k}^{u}\left(\bar{\gamma}_{k}^{u} \sum_{s=1}^{p} \mathfrak{C}_{s, k}\right)^{T} \\
& \times\left\{\bar{\gamma}_{k}^{u} \sum_{m=1}^{p} \sum_{n=1}^{p} \mathfrak{C}_{m, k} M_{k}^{u}\left(\bar{\gamma}_{k}^{u} \mathfrak{C}_{n, k}\right)^{T}\right. \\
& +\bar{\gamma}_{k}^{u} \sum_{m=1}^{p} \sum_{n=1}^{p} \Gamma_{m, \hbar_{k}} \tilde{\Lambda}_{m, k} \mathcal{C}_{m, k} P_{\xi_{k}}\left(\bar{\gamma}_{k}^{u} \Gamma_{n, \hbar_{k}} \mathcal{C}_{n, k}\right)^{T} \\
& \left.+\mathcal{R}_{k}\left[1-\bar{\varphi}_{k}^{u}\right]\right\}^{-1} \bar{\gamma}_{k}^{u} \sum_{t=1}^{p} \mathfrak{C}_{t, k} M_{k}^{u} \mathcal{A}_{k}^{T},
\end{aligned}
$$

which is exactly the same as (28). Assuming that the initial condition of (28) is independent of the realization in regard to the censoring sequence $\gamma_{1: k}$, we set $M_{0}^{u}=M_{0}>0$. For $k>0$, since the performance of the optimal protocol-based TKF must be no less than any of its suboptimal counterparts, it can be concluded that $M_{k} \leq M_{k}^{u}$. As such, $M_{k}^{u}$ is an upper bound on $M_{k}$ for all $k \geq 0$. This completes the proof.

\section{Proof of Theorem 4}

Proof: Denoting $\mathfrak{A}_{k} \triangleq \mathcal{A}_{k}+\gamma_{k} \mathfrak{K}_{k} \sum_{m=1}^{p} \mathfrak{C}_{m, k}$ and $\mathfrak{K}_{k} \triangleq$ $-\mathcal{A}_{k} K_{k}$, we have

$$
\begin{aligned}
& \mathfrak{A}_{k} P_{\tilde{\xi}_{k}^{-}}\left(\gamma_{k} \sum_{m=1}^{p} \mathfrak{C}_{m, k}\right)^{T}+\mathfrak{K}_{k}\left\{\gamma_{k} \sum_{m=1}^{p} \sum_{n=1}^{p} \Gamma_{m, \hbar_{k}} \tilde{\Lambda}_{m, k}\right. \\
& \left.\quad \times \mathcal{C}_{m, k} P_{\xi_{k}}\left(\gamma_{k} \Gamma_{n, \hbar_{k}} \mathcal{C}_{n, k}\right)^{T}+\mathcal{R}_{k}\left[1-\bar{\varphi}_{k}\right]\right\} \\
& =\left(\mathcal{A}_{k}+\gamma_{k} \mathfrak{K}_{k} \sum_{m=1}^{p} \mathfrak{C}_{m, k}\right) P_{\tilde{\xi}_{k}^{-}}\left(\gamma_{k} \sum_{n=1}^{p} \mathfrak{C}_{n, k}\right)^{T} \\
& +\mathfrak{K}_{k}\left\{\gamma_{k} \sum_{m=1}^{p} \sum_{n=1}^{p} \Gamma_{m, \hbar_{k}} \tilde{\Lambda}_{m, k} \mathcal{C}_{m, k} P_{\xi_{k}}\left(\gamma_{k} \Gamma_{n, \hbar_{k}} \mathcal{C}_{n, k}\right)^{T}\right. \\
& \left.+\mathcal{R}_{k}\left[1-\bar{\varphi}_{k}\right]\right\} \\
& =\mathcal{A}_{k} P_{\tilde{\xi}_{k}^{-}}\left(\gamma_{k} \sum_{n=1}^{p} \mathfrak{C}_{n, k}\right)^{T}+\mathfrak{K}_{k} P_{\tilde{y}_{k}^{-}} \\
& =0 .
\end{aligned}
$$

It follows from (26) that

$$
\begin{aligned}
P_{\tilde{\xi}_{k+1}^{-}}= & \left(1-\gamma_{k}\right)\left(\mathcal{A}_{k} P_{\tilde{\xi}_{k}^{-}} \mathcal{A}_{k}^{T}+\mathcal{B}_{k} Q_{k} \mathcal{B}_{k}^{T}\right) \\
& +\gamma_{k}\left(\mathcal{A}_{k} P_{\tilde{\xi}_{k}^{-}} \mathcal{A}_{k}^{T}+\mathcal{B}_{k} Q_{k} \mathcal{B}_{k}^{T}\right)-\mathcal{A}_{k} P_{\tilde{\xi}_{k}}
\end{aligned}
$$




$$
\begin{aligned}
& \times\left(\gamma_{k} \sum_{m=1}^{p} \mathfrak{C}_{m, k}\right)^{T} P_{\tilde{y}_{k}^{-}}^{-1} \gamma_{k} \sum_{n=1}^{p} \mathfrak{C}_{n, k} P_{\tilde{\xi}_{k}^{-}} \mathcal{A}_{k}^{T} \\
= & \left(1-\gamma_{k}\right)\left(\mathcal{A}_{k} P_{\tilde{\xi}_{k}^{-}} \mathcal{A}_{k}^{T}+\mathcal{B}_{k} Q_{k} \mathcal{B}_{k}^{T}\right) \\
& +\gamma_{k}\left(\mathcal{A}_{k} P_{\tilde{\xi}_{k}^{-}} \mathcal{A}_{k}^{T}+\mathcal{B}_{k} Q_{k} \mathcal{B}_{k}^{T}\right) \\
& +\gamma_{k} \mathfrak{K}_{k} \sum_{m=1}^{p} \mathfrak{C}_{m, k} P_{\tilde{\xi}_{k}^{-}} \mathcal{A}_{k}^{T} \\
= & \left(1-\gamma_{k}\right)\left(\mathcal{A}_{k} P_{\tilde{\xi}_{k}^{-}} \mathcal{A}_{k}^{T}+\mathcal{B}_{k} Q_{k} \mathcal{B}_{k}^{T}\right) \\
& +\gamma_{k}\left(\mathfrak{A}_{k} P_{\tilde{\xi}_{k}^{-}} \mathcal{A}_{k}^{T}+\mathcal{B}_{k} Q_{k} \mathcal{B}_{k}^{T}\right) .
\end{aligned}
$$

Inserting (42) into (43) generates

$$
\begin{aligned}
P_{\tilde{\xi}_{k+1}^{-}}= & \left(1-\gamma_{k}\right)\left(\mathcal{A}_{k} P_{\tilde{\xi}_{k}^{-}} \mathcal{A}_{k}^{T}+\mathcal{B}_{k} Q_{k} \mathcal{B}_{k}^{T}\right) \\
& +\gamma_{k}\left(\mathfrak{A}_{k} P_{\tilde{\xi}_{k}^{-}} \mathcal{A}_{k}^{T}+\mathcal{B}_{k} Q_{k} \mathcal{B}_{k}^{T}\right) \\
& +\gamma_{k} \mathfrak{A}_{k} P_{\tilde{\xi}_{k}^{-}}\left(\gamma_{k} \sum_{m=1}^{p} \mathfrak{C}_{m, k}\right)^{T} \mathfrak{K}_{k}^{T} \\
& +\gamma_{k} \mathfrak{K}_{k}\left\{\gamma_{k} \sum_{m=1}^{p} \sum_{n=1}^{p} \Gamma_{m, \hbar_{k}} \tilde{\Lambda}_{m, k} \mathcal{C}_{m, k}\right. \\
& \left.\times P_{\xi_{k}}\left(\gamma_{k} \Gamma_{n, \hbar_{k}} \mathcal{C}_{n, k}\right)^{T}+\mathcal{R}_{k}\left[1-\bar{\varphi}_{k}\right]\right\} \mathfrak{K}_{k}^{T} \\
= & \left(1-\gamma_{k}\right)\left(\mathcal{A}_{k} P_{\tilde{\xi}_{k}^{-}} \mathcal{A}_{k}^{T}+\mathcal{B}_{k} Q_{k} \mathcal{B}_{k}^{T}\right) \\
& +\gamma_{k}\left(\mathfrak{A}_{k} P_{\tilde{\xi}_{k}^{-}} \mathfrak{A}_{k}^{T}+\mathcal{B}_{k} Q_{k} \mathcal{B}_{k}^{T}\right) \\
& +\gamma_{k} \mathfrak{K}_{k}\left\{\gamma_{k} \sum_{m=1}^{p} \sum_{n=1}^{p} \Gamma_{m, \hbar_{k}} \tilde{\Lambda}_{m, k} \mathcal{C}_{m, k}\right. \\
& \left.\times P_{\xi_{k}}\left(\gamma_{k} \Gamma_{n, \hbar_{k}} \mathcal{C}_{n, k}\right)^{T}+\mathcal{R}_{k}\left[1-\bar{\varphi}_{k}\right]\right\} \mathfrak{K}_{k}^{T} .
\end{aligned}
$$

Taking expectation on both sides of (44) and noting the non-negative definiteness of $P_{\tilde{\xi}_{k}^{-}}, P_{\xi_{k}}, \tilde{\Lambda}_{m, k}$ and $\mathcal{R}_{k}$ lead to

$$
\begin{aligned}
M_{k+1}= & \mathbb{E}\left\{\left(1-\gamma_{k}\right)\left(\mathcal{A}_{k} P_{\tilde{\xi}_{k}} \mathcal{A}_{k}^{T}+\mathcal{B}_{k} Q_{k} \mathcal{B}_{k}^{T}\right)\right\} \\
& +\mathbb{E}\left\{\gamma_{k}\left(\mathfrak{A}_{k} P_{\tilde{\xi}_{k}^{-}} \mathfrak{A}_{k}^{T}+\mathcal{B}_{k} Q_{k} \mathcal{B}_{k}^{T}\right)\right\} \\
& +\mathbb{E}\left\{\gamma _ { k } \mathfrak { K } _ { k } \left\{\gamma_{k} \sum_{m=1}^{p} \sum_{n=1}^{p} \Gamma_{m, \hbar_{k}} \tilde{\Lambda}_{m, k} \mathcal{C}_{m, k}\right.\right. \\
& \left.\left.\times P_{\xi_{k}}\left(\gamma_{k} \Gamma_{n, \hbar_{k}} \mathcal{C}_{n, k}\right)^{T}+\mathcal{R}_{k}\left[1-\bar{\varphi}_{k}\right]\right\} \mathfrak{K}_{k}^{T}\right\} \\
\geq & \mathbb{E}\left\{\left(1-\gamma_{k}\right)\left(\mathcal{A}_{k} P_{\tilde{\xi}_{k}} \mathcal{A}_{k}^{T}+\mathcal{B}_{k} Q_{k} \mathcal{B}_{k}^{T}\right)\right\} \\
& +\mathbb{E}\left\{\gamma_{k} \mathcal{B}_{k} Q_{k} \mathcal{B}_{k}^{T}\right\} \\
= & \left(1-\bar{\gamma}_{k}\right) \mathcal{A}_{k} M_{k} \mathcal{A}_{k}^{T}+\bar{\gamma}_{k} \mathcal{B}_{k} Q_{k} \mathcal{B}_{k}^{T} .
\end{aligned}
$$

Inspired by (45), let us define

$$
M_{k+1}^{l} \triangleq\left(1-\bar{\gamma}_{k}\right) \mathcal{A}_{k} M_{k}^{l} \mathcal{A}_{k}^{T}+\bar{\gamma}_{k} \mathcal{B}_{k} Q_{k} \mathcal{B}_{k}^{T},
$$

which is initialized at $M_{0}^{l}=0$. Now, let us prove $M_{k}^{l} \leq M_{k}$ via mathematical induction. Noticeably, at the initial time $k=$
0 , we have $M_{0}^{l}=0 \leq M_{0}$. Supposing that $M_{k}^{l} \leq M_{k}$ holds at time $k$, we have

$$
\begin{aligned}
M_{k+1}^{l} & =\left(1-\bar{\gamma}_{k}\right) \mathcal{A}_{k} M_{k}^{l} \mathcal{A}_{k}^{T}+\bar{\gamma}_{k} \mathcal{B}_{k} Q_{k} \mathcal{B}_{k}^{T} \\
& \leq\left(1-\bar{\gamma}_{k}\right) \mathcal{A}_{k} M_{k} \mathcal{A}_{k}^{T}+\bar{\gamma}_{k} \mathcal{B}_{k} Q_{k} \mathcal{B}_{k}^{T} \\
& \leq M_{k+1},
\end{aligned}
$$

where the last inequality holds from (45). As a result, it can be concluded that, $M_{k}^{l} \leq M_{k}$ holds for all $k \geq 0$, i.e. $M_{k}^{l}$ is a lower bound on $M_{k}$. This completes the proof.

\section{REFERENCES}

[1] A. Assa, and F. Janabi-Sharifi, A robust vision-based sensor fusion approach for real-time pose estimation, IEEE Transactions on Cybernetics, vol. 44, no. 2, pp. 217-227, 2014.

[2] B. Allik, C. Miller, M. J. Piovoso, and R. Zurakowski, The Tobit Kalman filter: an estimator for censored measurements, IEEE Transactions on Control Systems Technology, vol. 24, no. 1, pp. 365-371, 2016.

[3] B. D. O. Anderson, and J. B. Moore, Optimal Filtering. Englewood Cliffs, NJ, USA: Prentice-Hall, 1979.

[4] S. Appadwedula, V. V. Veeravalli, and D. L. Jones, Decentralized detection with censoring sensors, IEEE Transactions on Signal Processing, vol. 56, no. 4, pp. 1362-1373, 2008.

[5] R. Caballero-Águila, A. Hermoso-Carazo, and J. Linares-Pérez, Distributed fusion filters from uncertain measured outputs in sensor networks with random packet losses, Information Fusion, vol. 34, pp. 7079, 2017.

[6] P. Casolia, N. Authiera, X. Jacqueta, and J. Cartier, Characterization of the caliban and prospero critical assemblies neutron spectra for integral measurements experiments, Nuclear Data Sheets, vol. 118, pp. 554-557, 2014.

[7] R. Carson, and Y. Sun, The Tobit model with a non-zero threshold, The Econometrics Journal, vol. 10, no. 3, pp. 488-502, 2007.

[8] Y. Chen, Z. Wang, Y. Yuan and P. Date, Distributed $H_{\infty}$ filtering for switched stochastic delayed systems over sensor networks with fading measurements, IEEE Transactions on Cybernetics, vol. 50, no. 1, pp. 214, Jan. 2020.

[9] Y. Chen, Z. Wang, L. Wang and W. Sheng, Finite-horizon $H_{\infty}$ state estimation for stochastic coupled networks with random inner couplings using Round-Robin protocol, IEEE Transactions on Cybernetics, in press, DOI: 10.1109/TCYB.2020.3004288.

[10] Y. Chen, Z. Wang, B. Shen and H. Dong, Exponential synchronization for delayed dynamical networks via intermittent control: Dealing with actuator saturations, IEEE Transactions on Neural Networks and Learning Systems, vol. 30, no. 4, pp. 1000-1012, Apr. 2019.

[11] D. Ciuonzo, A. Aubry, and V. Carotenuto, Rician MIMO channel- and jamming-aware decision fusion, IEEE Transactions on Signal Processing, vol. 65, no. 15, pp. 3866-3880, 2017.

[12] Z. Du, and X. Li, Strong tracking Tobit Kalman filter with model uncertainties, International Journal of Control, Automation and Systems, vol. 17, no. 2, pp. 345-355, 2019.

[13] A. Gopalakrishnan, N. S. Kaisare, and S. Narasimhan, Incorporating delayed and infrequent measurements in extended Kalman filter based nonlinear state estimation, Journal of Process Control, vol. 21, no. 1, pp. 119-129, 2011.

[14] H. Geng, Z. Wang, Y. Cheng, F. E. Alsaadi, and A. M. Dobaie, State estimation under non-gaussian Lévy and time-correlated additive sensor noises: a modified Tobit Kalman filtering approach, Signal Processing, vol. 154, pp. 120-128, 2019.

[15] H. Geng, Z. Wang, Y. Liang, Y. Cheng, and F. E. Alsaadi, Tobit Kalman filter with time-correlated multiplicative sensor noises under redundant channel transmission, IEEE Sensors Journal, vol. 17, no. 24, pp. 83678377, 2017.

[16] H. Geng, Z. Wang, and Y. Cheng, Distributed federated Tobit Kalman filter fusion over a packet-delaying network: a probabilistic perspective, IEEE Transactions on Signal Processing, vol. 66, no. 17, pp. 4477-4489, 2018.

[17] Y. Guo, and B. Huang, State estimation incorporating infrequent, delayed and integral measurements, Automatica, vol. 58, pp. 32-38, 2015.

[18] J. Hampshire, and J. Strohbehn, Tobit maximum-likelihood estimation for stochastic time series affected by receiver saturation, IEEE Transactions on Information Theory, vol. 38, no. 2, pp. 457-469, 1992. 
[19] F. Han, G. Wei, D. Ding and Y. Song, Local condition based consensus filtering with stochastic nonlinearities and multiple missing measurements, IEEE Transactions on Automatic Control, vol. 62, no. 9, pp. 4784-4790, Sep. 2017.

[20] J. Hu, Z. Wang, G.-P. Liu and H. Zhang, Variance-constrained recursive state estimation for time-varying complex networks with quantized measurements and uncertain inner coupling, IEEE Transactions on Neural Networks and Learning Systems, vol. 31, no. 6, pp. 1955-1967, Jun. 2020.

[21] J. Huang, and X. He, Detection of intermittent fault for discretetime systems with output dead-zone: a variant Tobit Kalman filtering approach, Journal of Control Science and Engineering, vol. 2017, Article ID 7849841, 9 pages, 2017.

[22] Q. Li, Z. Wang, N. Li and W. Sheng, A dynamic event-triggered approach to recursive filtering for complex networks with switching topologies subject to random sensor failures, IEEE Transactions on Neural Networks and Learning Systems, in press, DOI: 10.1109/TNNLS.2019.2951948

[23] W. Li, Y. Jia, and J. Du, Tobit Kalman filter with time-correlated multiplicative measurement noise, IET Control Theory \& Applications, vol. 11, no. 1, pp. 122-128, 2017.

[24] W. Li, Z. Wang, Q. Liu and L. Guo, An information aware eventtriggered scheme for particle filter based remote state estimation, Automatica, vol. 103, pp. 151-158, May 2019.

[25] X. Li, F. Han, N. Hou, H. Dong and H. Liu, Set-membership filtering for piecewise linear systems with censored measurements under RoundRobin protocol, International Journal of Systems Science, in press, DOI: 10.1080/00207721.2020.1768453.

[26] D. Liu, Z. Wang, Y. Liu and F. E. Alsaadi, Recursive resilient filtering for nonlinear stochastic systems with packet disorders, Journal of The Franklin Institute, vol. 357, no. 8, pp. 4817-4833, May 2020.

[27] Q. Liu, Z. Wang, X. He, and D. H. Zhou, Event-triggered resilient filtering with measurement quantization and random sensor failures: monotonicity and convergence, Automatica, vol. 94, pp. 458-464, 2018.

[28] Q. Liu, Z. Wang, Q.-L. Han and C. Jiang, Quadratic estimation for discrete time-varying non-Gaussian systems with multiplicative noises and quantization effects, Automatica, vol. 113, Art. no. 108714, Mar. 2020

[29] S. Liu, Z. Wang, G. Wei, and M. Li, Distributed set-membership filtering for multirate systems under the Round-Robin scheduling over sensor networks, IEEE Transactions on Cybernetics, to be published, 10.1109/TCYB.2018.2885653.

[30] Y. Liu, Z. Wang, and D. H. Zhou, State estimation and fault reconstruction with integral measurements under partially decoupled disturbances, IET Control Theory \& Applications, vol. 12, no. 10, pp. 1520-1526, 2018.

[31] Y. Liu, B. Shen and H. Shu, Finite-time resilient $H_{\infty}$ state estimation for discrete-time delayed neural networks under dynamic event-triggered mechanism, Neural Networks, vol. 121, pp. 356-365, Jan. 2020.

[32] Y. Liu, B. Shen and Q. Li, State estimation for neural networks with Markov-based nonuniform sampling: The partly unknown transition probability case, Neurocomputing, vol. 357, pp. 261-270, Sept. 2019.

[33] Y. Liu, Z. Wang, Y. Yuan and W. Liu, Event-triggered partial-nodesbased state estimation for delayed complex networks with bounded distributed delays, IEEE Transactions on Systems, Man, and CyberneticsSystems, vol. 49, no. 6, pp. 1088-1098, Jun. 2019.

[34] F. Yang, Q.-L. Han and Y. Liu, Distributed $H_{\infty}$ state estimation over a filtering network with time-varying and switching topology and partial information exchange, IEEE Transactions on Cybernetics, vol. 49, no. 3, pp. 870-882, Mar. 2019.

[35] J. Mao, D. Ding, G. Wei and H. Liu, Networked recursive filtering for time-delayed nonlinear stochastic systems with uniform quantisation under Round-Robin protocol, International Journal of Systems Science, vol. 50, no. 4, pp. 871-884, Mar. 2019.

[36] E. V. Markov, and O. M. Mikhailov, Integral measurements of the color of nanodimensional radiators, Measurement Techniques, vol. 57, no. 9, pp. 990-996, 2014.

[37] N. Nahi, Optimal recursive estimation with uncertain observation, IEEE Transactions on Information Theory, vol. 15, no. 4, pp. 457-462, 1969

[38] W. Qian, Y. Li, Y. Chen, and W. Liu, $L_{2}-L_{\infty}$ filtering for stochastic delayed systems with randomly occurring nonlinearities and sensor saturation, International Journal of Systems Science, vol. 51, no. 13, pp. 2360-2377, Oct. 2020.

[39] D. Spinello, and D. J. Stilwell, Nonlinear estimation with statedependent Gaussian observation noise, IEEE Transactions on Automatic Control, vol. 55, no. 6, pp. 1358-1366, 2010.

[40] B. Shen, Z. Wang, D. Wang and H. Liu, Distributed state-saturated recursive filtering over sensor networks under Round-Robin protocol,
IEEE Transactions on Cybernetics, vol. 50, no. 8, pp. 3605-3615, Aug. 2020.

[41] Y. Shen, Z. Wang, B. Shen, F. E. Alsaadi, and F. E. Alsaadi, Fusion estimation for multi-rate linear repetitive processes under weighted tryonce-discard protocol, Information Fusion, vol. 55, pp. 281-291, 2020.

[42] J. Tobin, Estimation of relationships for limited dependent variables, Econometrica, vol. 26, no. 1, pp. 24-36, 1958.

[43] S. Tripathi, M. Gehlot, J. Hussain, G. Mishra, V. Kumar, and S. Chouksey, Field integral measurement of a six period undulator in a pulsed wire set up, Optics Communications, vol. 284, no. 1, pp. 350357, 2011.

[44] X. Wan, Z. Wang, M. Wu and X. Liu, $H_{\infty}$ state estimation for discretetime nonlinear singularly perturbed complex networks under the RoundRobin protocol, IEEE Transactions on Neural Networks and Learning Systems, vol. 30, no. 2, pp. 415-426, Feb. 2019.

[45] Y. Zhang, Z. Wang, and L. Ma, Variance-constrained state estimation for networked multi-rate systems with measurement quantization and probabilistic sensor failures, International Journal of Robust and Nonlinear Control, vol. 26, pp. 3507-3523, 2016.

[46] D. Zhao, Z. Wang, Y. Chen and G. Wei, Proportional-integral observer design for multi-delayed sensor-saturated recurrent neural networks: A dynamic event-triggered protocol, IEEE Transactions on Cybernetics, vol. 50, no. 11, pp. 4619-4632, Nov. 2020.

[47] Z. Zhao, Z. Wang, L. Zou and J. Guo, Set-Membership filtering for timevarying complex networks with uniform quantisations over randomly delayed redundant channels, International Journal of Systems Science, in press, DOI: $10.1080 / 00207721.2020 .1814898$.

[48] L. Zou, Z. Wang, Q.-L. Han and D. H. Zhou, Full information estimation for time-varying systems subject to Round-Robin scheduling: A recursive filter approach, IEEE Transactions on Systems, Man, and Cybernetics-Systems, in press, DOI: 10.1109/TSMC.2019.2907620.

[49] L. Zou, Z. Wang, Q.-L. Han and D. H. Zhou, Moving horizon estimation of networked nonlinear systems with random access protocol, IEEE Transactions on Systems, Man, and Cybernetics-Systems, in press, DOI: 10.1109/TSMC.2019.2918002.

[50] L. Zou, Z. Wang, J. Hu and D. H. Zhou, Moving horizon estimation with unknown inputs under dynamic quantization effects, IEEE Transactions on Automatic Control, vol. 65, no. 12, pp. 5368-5375, Dec. 2020.

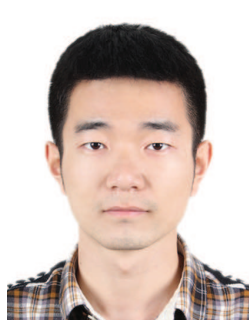

Hang Geng received the B.Sc. degree in electronic information engineering in 2011 from Civil Aviation Flight University of China, Guanghan, China, and the $\mathrm{Ph} . \mathrm{D}$. degree in control science and engineering in 2017 from Northwestern Polytechnical University, Xi'an, China.

$\mathrm{He}$ is currently an Associate Professor in the School of Automation Engineering, University of Electronic Science and Technology of China, Chengdu, China. From 2015 to 2016, he was a visiting $\mathrm{Ph} . \mathrm{D}$. student with the Department of Computer Science, Brunel University London, Uxbridge, U.K. From 2018 to 2019, he was a Post-Doctoral Fellow with the Department of Mechanical Engineering, University of Kansas, Lawrence, U.S. From 2019 to 2020, he was a Research Fellow with the Department of Computer Science, Brunel University London, Uxbridge, U.K. His research interests include information fusion, fault diagnosis and state estimation. 
This article has been accepted for publication in a future issue of this journal, but has not been fully edited. Content may change prior to final publication. Citation information: DOI10.1109/TSP.2020.3048245, IEEE Transactions on Signal Processing

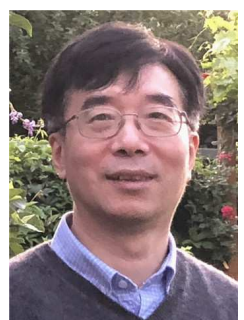

Zidong Wang (SM'03-F'14) was born in Jiangsu, China, in 1966. He received the B.Sc. degree in mathematics in 1986 from Suzhou University, Suzhou, China, and the M.Sc. degree in applied mathematics in 1990 and the Ph.D. degree in electrical engineering in 1994, both from Nanjing University of Science and Technology, Nanjing, China.

$\mathrm{He}$ is currently Professor of Dynamical Systems and Computing in the Department of Computer Science, Brunel University London, U.K. From 1990 to 2002, he held teaching and research appointments in universities in China, Germany and the UK. Prof. Wang's research interests include dynamical systems, signal processing, bioinformatics, control theory and applications. He has published more than 600 papers in international journals. He is a holder of the Alexander von Humboldt Research Fellowship of Germany, the JSPS Research Fellowship of Japan, William Mong Visiting Research Fellowship of Hong Kong.

Prof. Wang serves (or has served) as the Editor-in-Chief for International Journal of Systems Science, the Editor-in-Chief for Neurocomputing, and an Associate Editor for 12 international journals including IEEE Transactions on Automatic Control, IEEE Transactions on Control Systems Technology, IEEE Transactions on Neural Networks, IEEE Transactions on Signal Processing, and IEEE Transactions on Systems, Man, and Cybernetics-Part C. He is a Member of the Academia Europaea, a Fellow of the IEEE, a Fellow of the Royal Statistical Society and a member of program committee for many international conferences.

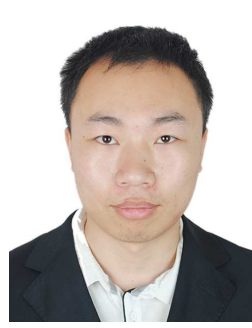

Lei Zou (SM'20) received the B.Sc. degree in automation from Beijing Institute of Petrochemical Technology, Beijing, China, in 2008, the M.Sc. degree in control science and engineering from China University of Petroleum (Beijing Campus), Beijing, China, in 2011 and the Ph.D degree in control science and engineering in 2016 from Harbin Institute of Technology, Harbin, China. From October 2013 to October 2015, he was a visiting Ph.D. student with the Department of Computer Science, Brunel University London, Uxbridge, U.K.

Since 2019, he has been working as a Research Fellow with the Department of Computer Science, Brunel University London, Uxbridge, Uxbridge, U. $\mathrm{K}$. His research interests include control and filtering of networked systems, moving-horizon estimation, and state estimation subject to outliers.

Dr. Zou is currently serving as an Associate Editor for Neurocomputing and International Journal of Systems Science; a Senior Member of IEEE and a Member of Chinese Association of Automation; and a very active reviewer for many international journals.

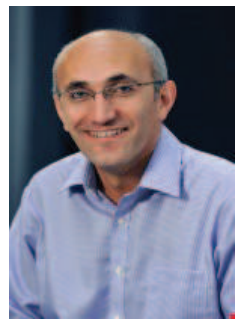

Alireza Mousavi (SM'13) received the Ph.D. degree in 2000 from the Department of Electronic and Computer Engineering, Brunel University London, Uxbridge, U.K.

He is currently a Reader of Systems Engineering and Computing with Brunel University London. His research interests include smart supervisory control and data acquisition systems applied to realtime systems modeling and optimization. The key areas of application are in stochastic modeling, ontology alignment, and sensor networks. Dr. Mousavi is a member of the Institute of Engineering and Technology, and a very active reviewer for many international journals.

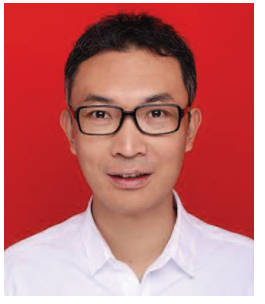

Yuhua Cheng (SM'13) received the Ph.D. degree in instrumentation science and technology in 2007 from Sichuan University, Chengdu, China.

$\mathrm{He}$ is currently a Professor of Instrument Science and Technology in the School of Automation Engineering, University of Electronic Science and Technology of China, Chengdu, China. From 2013 to 2014 , he was a Guest Researcher at the University of Toronto, Toronto, Canada. His research interests include electronic instrumentation, imaging/sensing for nondestructive evaluation, intelligent prognostics and health management of complex systems, and numerical modeling and simulation. Prof. Cheng is currently serving as an Associate Editor for Neurocomputing and IEEE Transactions on Instrumentation and Measurement, and a very active reviewer for many international journals. 